\title{
LIDAR-Assisted Exact Output Regulation for Load Mitigation in Wind Turbines
}

\author{
Amin Mahdizadeh, Robert Schmid and Denny Oetomo*
}

October 18, 2019

\begin{abstract}
Optimising wind turbine performance involves maximising energy harvesting while seeking to minimise load fatigues on the tower structure, blades and rotor. The problem is inherently difficult due to the slow response of wind turbines compared to wind variation frequencies. To improve turbine control performance, wind preview measurement technologies such as Light Detection And Ranging (LIDAR) have been a point of interest for researchers in recent years. However, the effective augmentation of wind preview information into control methodologies has to date proven to be a challenging problem. In this paper, we explore the application of a classical control methodology known as Exact Output Regulation (EOR) for improving the control performance of a LIDAR-enhanced wind turbine. The EOR controller is designed to achieve the rejection of known input disturbances, while also ensuring the system output tracks a desired reference signal. The controller is comprised of a state feedback controller together with a feedforward gain. The LIDAR wind preview information is used to obtain a low-order exosystem for modeling wind dynamics. This wind exosystem is used to obtain the feedforward gain matrix that enables the EOR controller to effectively reject the input disturbance and achieve the desired reference tracking. Extensive simulations of the EOR controller with a broad range of wind speeds in both partial load and full load operating regions are performed on the full nonlinear aero-elastic model of the National Renewable Energy Laboratory (NREL) $5 \mathrm{MW}$ reference wind turbine. For performance comparisons, we also implement a Baseline torque controller and a commonly used feedforward control method known as Disturbance Accommodation Control (DAC). The results show that, in comparison with a Baseline and DAC controller, the EOR controller can provide substantially improved reduction of fatigue loads and smoother power output, without compromising energy production levels.
\end{abstract}

\section{Introduction}

Reliable power production from wind is a difficult problem, due to the intermittent nature of the wind. It has been the subject of research interest from the early days of electrical wind turbines. The transformation of the free kinetic energy of the wind into mechanical,

*A. Mahdizadeh and R. Schmid are with the Department of Electrical and Electronic Engineering, University of Melbourne, 3010 Australia. D. Oetomo is with the Department of Mechanical Engineering, University of Melbourne, 3010 Australia. email: amahdizadeh@student.unimelb.edu.au,rschmid@unimelb.edu.au, doetomo@unimelb.edu.au 
and subsequently, electrical energy comes with the cost of the structure and materials of the turbine as well their maintenance. Two simultaneous approaches taken to reduce the levelised cost of wind energy (LCOE) [1] are maximizing the energy harvesting efficiency and reducing the cost of maintenance by reducing turbine fatigue loads. From the control engineering point of view, performance improvements may be achieved through a combination of additional measurements and superior control methodologies.

Mechanical loads on the wind turbine structure induced by sudden variations of the wind can be mitigated by the control of the blade pitch angle and generator torque control [2]. Energy harvesting efficiency can be improved by more precisely steering the wind turbine states on their optimal trajectories. The use of feedback control is the conventional approach for stabilizing and regulating dynamical systems, however, feedback control for turbines may not yield satisfactory system behaviour, as it assumes the turbine only reacts to the variations of the wind which may have already influenced the system states and driven them away from their desired values. To address this shortcoming, Light Detection And Ranging (LIDAR) has been proposed as a new technology to provide estimates of upcoming wind speeds prior to the wind interacting with the turbine blades [3]. Recent LIDAR cost reductions have opened a new research area on the use of feedforward control for large scale wind turbines, using nacellebased LIDAR systems to obtain real-time wind speed and direction information up to several hundred meters ahead of the wind turbine [4,5]. Recently [6] collected extensive field data which showed that profiling LIDARs can measure wind speeds and turbulence intensities with acceptable accuracy, in comparison with tower-mounted cup and sonic anemometers.

An early work involving LIDAR for feedforward control of the wind turbines [7] showed that an augmented LIDAR feedforward control may improve turbine energy harvesting and load reduction. Further investigations using non-causal series expansion model-inverse method appeared in [8], where it was shown that a lower damage equivalent load (DEL) on tower root fore-aft oscillations could be obtained relative to a Baseline controller, with no loss in the produced power. In [9], a preview-based feedforward method assuming highly idealized wind measurements concluded that wind evolutions in more realistic conditions can eliminate advantages gained by using preview-based feedforward techniques. The LIDAR-assisted design in [10] used three different model inversion methods to augment the feedback loop: the nonminimum-phase zeros ignore (NPZ-Ignore), the zero-phase-error tracking controller (ZPETC) and the zero-magnitude-error tracking controller (ZMETC); some improvement in some of the loads were obtained. Two early field testing surveys of LIDAR-based feedforward control using model inversion methods were carried out in [11, 12]. The results showed evidence of tower load reduction by $10 \%$ due to the utilization of LIDAR, confirming the previous results on simulations. In [13] load reductions were improved by using Continuous-Wave LIDAR. Although model inversion methods are feasible in the presence of look-ahead LIDAR information, they require the use of approximated models of the plant inverse to avoid the effects of non-minimum phase zero inversion.

LIDAR-assisted control has also been tested for improving energy harvesting at below rated wind speeds. Results from [14 showed that LIDAR-aided rotor speed and yaw angle control yielded increased energy production. Field tests of the methods proposed in [14 were extended in [15] with real data collected from LIDAR where $0.3 \%$ improvement in energy gain was achieved, however this came at the cost of doubling the loads on the shaft. In [4] three different methods of wind turbine control are augmented by LIDAR and compared at below-rated wind speeds: the optimally tracking rotor (OTR) control scheme [16], Preview Control [17] and Disturbance Accommodation Control (DAC), sometimes also known as Disturbance Tracking 
Control (DTC) [18]. However, these methods were only able to increase energy harvesting by very small amounts, and these improvements came at the cost of substantial increases in some fatigue loads.

DAC has been one of the most widely used feedforward methods for wind turbine control during the last decade, due to its simplicity and capacity to estimate the effective wind speed on the rotor. It was first applied to wind turbines in [19], to counteract the effects of wind disturbances. Later, DAC methods have been used in [20] and [21] to reduce blade fatigue loads induced by the wind disturbances. Also in [22] it was applied for canceling asymmetric blade mass effects of a two-bladed wind turbine causing periodic loads. A field test on the Controls Advanced Research Turbine (CART) [23] showed that, compared to a Baseline PI controller, DAC can reduce structural dynamic loads in real case scenarios.

Efforts have been made to enhance DAC by augmenting the controller with LIDAR information, replacing the estimated wind speed with the LIDAR measured speed. However, to date only modest performance improvements have been achieved. [4] showed that the LIDAR augmented DAC (known as DAC+LIDAR) achieved less than $0.5 \%$ improvement in power production, and this improvement came at the cost of a $5.7 \%$ increase in rotor fatigue load.

Model Predictive Control (MPC) methods have also been a point of interest since they can readily accommodate LIDAR wind information. The simulation studies [24, 25] showed that nonlinear MPC can reduce structural loads for turbulent winds and extreme loads gusts. However, a key limitation of nonlinear MPC is the substantial computational cost, making it infeasible for real-time applications on conventional industry grade controllers.

In this paper, we present a novel method for turbine control that can make effective use of LIDAR information, without requiring the excessive computational costs of MPC. Our approach will employ the Exact Output Regulation (EOR) control methodology, and our results will show that it can deliver substantial reductions in fatigue loads, without compromising energy harvesting. Additionally, its rapid computation time makes it feasible for real-time implementation [26].

The EOR control methodology has played a central role in modern control systems design for several decades [27]- [28]. The output regulation problem considers a linear time invariant (LTI) plant that is assumed to be subject to known input time-varying disturbances, and whose output is desired to track a known time-varying reference signal. The reference signals and external disturbances are modelled as the outputs of a linear exosystem. Solution of the problem requires the design a combined state feedback and feedforward controller that will internally stabilise the plant while rejecting the disturbances and ensuring the output converges asymptotically to the desired reference signal. The required feedback and feedforward gain matrices are readily computable [28].

For turbine control, effective disturbance rejection involves the minimisation of wind disturbances on the control actuation. These are the rotor torque and also the blade pitch angle. Effective reference tracking involves operation of the turbine rotor and blades so as to generate the optimal energy from the wind. The problem is difficult as the wind frequency variation (turbulence) is much faster than the turbine response. The availability of LIDAR wind preview information enables the wind signal to be modeled as a low-order linear dynamical system. This linear system may then be incorporated into the EOR control methodology as an exosystem whose outputs provide the input disturbance and the output reference signal [29,30]. When the turbine is operating below its rated power, the reference signal is the value of the rotor speed that achieves the maximum power generation. When the turbine operates at its rated power, the reference is the value of the blade pitch angle that maintains the turbine at its rated 
power.

A simulation environment known as Turbine Output Regulation (TOR) has been developed by the authors to apply EOR to the control of a $5 M W$ reference Horizontal Axis Wind Turbine (HAWT) [31]. To simulate the turbine response, the Fatigue, Aerodynamics, Structures, and Turbulence (FAST) code using a high-order aero-elastic nonlinear turbine model developed by National Renewable Energy Laboratory (NREL) [32] will be used. The NREL TurbSim package [33] is used to simulate realistic wind fields, and damage equivalent loads (DELs) are computed by the Rain-Flow-Counting-Algorithm open source MATLAB ${ }^{\circledR}$ code [34]. For performance comparison purposes, TOR obtains a feedforward controller using the DAC method, and also a Baseline torque controller. Energy harvesting and DELs are computed within the TOR simulation environment to allow performance comparisons of these three controllers, for a broad range of wind speeds and intensities.

Our simulations studies on the $5 M W$ HAWT model show that, in comparison with DAC and the Baseline method, the EOR controller can substantially reduce the fatigue loads on the tower, blades and low speed shaft. Additionally, EOR is able to reduce the standard deviation of rotor speed and output power, without any loss in energy harvesting. The authors believe that EOR is able to obtain these improvements through its modeling of the wind dynamics. Where DAC treats the wind signal as a constant disturbance and does not consider any output tracking objective, EOR is able to accommodate derivatives of the wind signal, leading to improved tracking performance and disturbance rejection.

The remainder of this paper is organized as follows. Section 2 describes the turbine modelling in both rated and below rated operating regions. Section 3 introduces the control performance objectives and methods to be considered in our simulation studies. We provide a summary of the EOR control methodology for a general linear time invariant plant in state space form. The Baseline feedback controller and DAC control method are also described. In Section 4, we consider how to use raw LIDAR wind measurement data to develop a suitable low-order linear wind model that will be used as the exosystem for the development of an EOR controller for the turbine. Section 5 describes the turbine simulation environment used for our performance comparisons, and our simulation results will be presented and discussed in Section 6. Finally, in section 7 our conclusions and future work will be presented.

\section{Wind Turbine Modeling}

The wind turbine model used in this work is the NREL 5-MW reference Horizontal Axis Wind Turbine (HAWT) [31]. Detailed specifications of the turbine are presented in Table 1.

\section{$2.1 \quad$ Operating Regions}

Turbine operation may be divided into four distinct regions, determined by the mean wind speed, as depicted in Figure 1. The start-up region applies for very low wind speeds, where the kinetic energy of wind is insufficient for turbine operation. At the cut-in wind speed (about $3 \mathrm{~m} / \mathrm{s}$ ), the turbine begins to operate in Region 2. The higher the wind speed, the greater the energy that can be harvested by the blades. The rated power of the turbine is determined by factors such as the mechanical load capacity of the components as well as the limits on

the electrical power and currents deliverable by the generator. The wind speed at which the turbine is able to generate its rated power is called the rated wind speed, (about $11.4 \mathrm{~m} / \mathrm{s}$ ), 
Table 1: The NREL 5-MW Wind Turbine Specifications

\begin{tabular}{|c|c|c|}
\hline Quantity & Symbol & Value \\
\hline Rated power & $\bar{P} P_{\text {rated }}$ & $5 M W$ \\
\hline Rated rotor speed & $\Omega_{\text {rated }}$ & $12.1 \mathrm{rpm}$ \\
\hline Rated wind speed & $V_{\text {rated }}$ & $11.4 \mathrm{~m} / \mathrm{s}$ \\
\hline $\begin{array}{l}\text { Rated generator } \\
\text { torque }\end{array}$ & $M_{\text {rated }}$ & $43.1 \mathrm{kNm}$ \\
\hline Cut-in wind speed & $V_{\text {in }}$ & $3 \mathrm{~m} / \mathrm{s}$ \\
\hline Cut-out wind speed & $V_{\text {out }}$ & $25 \mathrm{~m} / \mathrm{s}$ \\
\hline Rotor radius & $R$ & $63 \mathrm{~m}$ \\
\hline Hub height & $h_{H}$ & $90 \mathrm{~m}$ \\
\hline $\begin{array}{l}\text { Rotor moment of iner- } \\
\text { tia }\end{array}$ & $J_{r}$ & $11.8 \mathrm{kt} / \mathrm{m}^{2}$ \\
\hline $\begin{array}{l}\text { Generator moment of } \\
\text { inertia }\end{array}$ & $J_{g}$ & $534 \mathrm{~kg} / \mathrm{m}^{2}$ \\
\hline Drive-train Stiffness & $K_{d}$ & $867 \mathrm{MNm} / \mathrm{rad}$ \\
\hline Drive-train Damping & $C_{d}$ & $6.2 \times 10^{6}$ \\
\hline Gearbox ratio & $i$ & $1 / 97$ \\
\hline $\begin{array}{l}\text { Tower equivalent } \\
\text { modal mass }\end{array}$ & $m_{T e}$ & $4.36 \times 10^{3} \mathrm{~kg}$ \\
\hline $\begin{array}{ll}\text { Tower } & \text { structural } \\
\text { damping } & \end{array}$ & $c_{T e}$ & 17782 \\
\hline Bending stiffness & $k_{T e}$ & $1.81 \mathrm{MN} / \mathrm{m}$ \\
\hline $\begin{array}{l}\text { Static tower-top dis- } \\
\text { placement in absence } \\
\text { of thrust forces }\end{array}$ & $x_{T 0}$ & $-0.0140 \mathrm{~m}$ \\
\hline $\begin{array}{l}\text { Blade pitch actuator } \\
\text { undamped natural fre- } \\
\text { quency }\end{array}$ & $\omega$ & $2 \pi \mathrm{rad} / \mathrm{s}$ \\
\hline $\begin{array}{l}\text { Blade pitch actuator } \\
\text { damping factor }\end{array}$ & $\zeta$ & 0.70 \\
\hline $\begin{array}{l}\text { Optimal tip speed ra- } \\
\text { tio }\end{array}$ & $\lambda^{*}$ & 7.55 \\
\hline $\begin{array}{l}\text { Maximum power coef- } \\
\text { ficient }\end{array}$ & $C_{p}^{*}$ & 0.482 \\
\hline
\end{tabular}




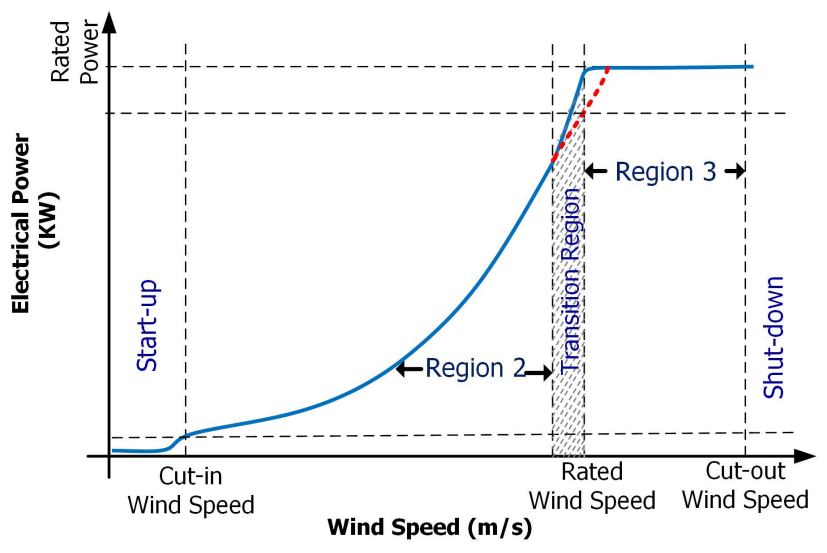

Figure 1: Wind Turbine Operating Regions 25$]$

and wind speeds above this are referred to as Region 3. The shut-down region applies when the wind speed exceeds a safe limit known as the cut-out wind speed (about $25 \mathrm{~m} / \mathrm{s}$ ).

\subsection{Optimal Power Generation}

The instantaneous power carried by the air moving through the vertical plane of the rotor blades is given by

$$
P_{\text {wind }}=\frac{1}{2} \rho A_{R} v_{\mathrm{x}}^{3}
$$

where $A_{R}$ is the swept area of the blades with radius $R, \rho$ is the air density and $v_{\mathrm{x}}$ is the magnitude of the component of the wind velocity vector that is perpendicular to the rotor plane. We refer to this perpendicular component as the longitudinal wind speed. The mechanical power extracted from the wind is $P_{\text {mech }}=\Omega_{r} M_{r}$, where $\Omega_{r}$ and $M_{r}$ are the rotor speed and rotor torque, respectively.

The blade tip speed ratio (TSR) is the ratio of the linear speed of the blade tip to the longitudinal wind speed $v_{\mathrm{x}}$ that can be formulated as:

$$
\lambda\left(\Omega_{r}, v_{\mathrm{x}}\right):=\frac{\Omega_{r} R}{v_{\mathrm{x}}} .
$$

This efficiency of the conversion of the wind energy to rotational-mechanical energy by the blades is the power coefficient of the turbine and is defined as

$$
C_{p}(\lambda, \theta):=\frac{P_{\text {mech }}}{P_{\text {wind }}}
$$

where $\theta$ is the blade pitch angle. According to Betz' law [35], the power coefficient is upper bounded $59.3 \%$. However, most wind turbines in real operating conditions fall short of this limit. The power coefficient is provided as a 2-D lookup table by the turbine manufacturer.

The aerodynamic torque of the rotor is modeled by the equation

$$
M_{a}\left(\Omega_{r}, v_{\mathrm{x}}, \theta\right):=\frac{1}{2} \rho \pi R^{3} \frac{C_{p}(\lambda, \theta)}{\lambda} v_{\mathrm{x}}^{2}
$$

In Region 2, the main objective of the turbine controller is to convert as much of the available wind energy into mechanical energy as possible. To achieve this, the blade pitch 
angle is kept at $\theta=0$ and a rotor torque controller is used to maintain the TSR at the optimal value $\lambda^{*}$ that maximizes $C_{p}$; thus

$$
C_{p}^{*}=C_{p}\left(\lambda^{*}, 0\right)
$$

Using (2), (5) and $\lambda^{*}$, we obtain $\Omega_{r}^{*}$, the rotor speed that yields optimal energy harvesting.

In Region 3, the convertible power $P_{\text {mech }}$ is larger than the wind turbine's rated power. Hence the maximum power extraction objective no longer applies, and the control objective becomes that of maintaining the turbine power output constant at the rated power level. This is achieved by keeping the generator torque at the rated value, and the blade pitch angle $\theta^{*}$ is chosen to ensure the aerodynamical torque of the rotor is at its rated value.

\subsection{Nonlinear Wind Turbine Model}

Wind turbines are nonlinear systems consisting of several dynamic components coupled together. In our simulations, we use the open source NREL FAST 7 code [32] to provide a high-fidelity turbine response simulation. However, a lower (reduced) order model is required

for the model-based controller design. The fifth-order nonlinear model from [36] describes the turbine drive-train as a two-mass system, and the blade pitch actuation system is modeled by a second order linear system with damping parameter $\zeta$ and natural frequency $\omega$ according to Table 1, This model is described as:

$$
\begin{aligned}
J_{r} \dot{\Omega}_{r} & =-C_{d}\left(\Omega_{r}-\Omega_{g}\right)-K_{d} \phi+M_{a}\left(\Omega_{r}, v_{\mathrm{x}}, \theta\right) \\
\dot{\phi} & =\Omega_{r}-\Omega_{g} \\
J_{g} \dot{\Omega}_{g} & =C_{d}\left(\Omega_{r}-\Omega_{g}\right)+K_{d} \phi-M_{g} \\
\ddot{\theta} & =-2 \zeta \omega \dot{\theta}+\omega^{2}\left(\theta_{c}-\theta\right)
\end{aligned}
$$

where $\Omega_{r}, \phi, \Omega_{g}$, and $\theta$ are the rotor speed, drive train torsion, generator speed and blade pitch angle, respectively. The generator torque $M_{g}$ and blade pitch command $\theta_{c}$ are the control inputs. Parameters $J_{r}$ and $J_{g}$ are the moments of inertia of the rotor and generator while $C_{d}$ and $K_{d}$ are the damping and stiffness coefficients of the drive train. It should be noted that in this work $\Omega_{g}$ has been normalized for the gearbox ratio $i$ so that it is in the same range as $\Omega_{r}$.

\subsection{Linearized Wind Turbine Model}

For the EOR and DAC controller design methods used in this study, a reduced order linearized model will be required. We follow the guidelines for turbine model linearization given in [20] and introduce $x=\left[\begin{array}{lllll}\Omega_{r} & \phi & \Omega_{g} & \theta & \dot{\theta}\end{array}\right]^{T}$ as the state variable vector. Also $u=\left[\begin{array}{ll}\theta_{c} & M_{g}\end{array}\right]^{T}$ is the control input vector, and $d=v_{\mathrm{x}}$ is the input disturbance. For a given mean wind speed, $v_{\mathrm{x}, 0}$, an equilibrium point $\left(x^{*}, u^{*}, d^{*}\right)$ may be found which satisfies

$$
\dot{x}=f\left(x^{*}, u^{*}, d^{*}\right)=0, \quad \text { subject to }: \Omega_{r}=\Omega_{r}^{*},
$$

where $\dot{x}=f(\cdot)$ describes the nonlinear dynamics (6)-(9). For mean wind speeds in Region 2, we have

$$
\Omega_{r}^{*}=\frac{\lambda^{*}}{R} v_{\mathrm{x}, 0}
$$

In Region $3, \Omega_{r}^{*}=\Omega_{\text {rated }}$, as given in Table 1 . 
Obtaining Jacobi matrices at the equilibrium point, we obtain the linear state space model in homogenised coordinates

$$
\Sigma:\left\{\begin{aligned}
\dot{\bar{x}}(t) & =A \bar{x}(t)+B \bar{u}(t)+H \bar{d}(t) \\
\bar{y}(t) & =C_{y} \bar{x}(t) \\
\bar{z}(t) & =C_{z} \bar{x}(t)
\end{aligned}\right.
$$

where $\bar{x}=x-x^{*}, \bar{u}=u-u^{*}$ and $\bar{d}=d-d^{*}$ represent coordinates homogenised to the equilibrium point. The homogenised measured output is denoted as $\bar{y}$, and $\bar{z}$ is the homogenised controlled output. The state matrices are

$$
\begin{gathered}
A=\left[\begin{array}{ccccc}
\frac{\left(\gamma-C_{d}\right)}{J_{r}} & \frac{-K_{d}}{J_{r}} & \frac{C_{d}}{J_{r}} & \frac{\beta}{J_{r}} & 0 \\
1 & 0 & -1 & 0 & 0 \\
\frac{C_{d}}{J g} & \frac{K_{d}}{J g} & \frac{-C_{d}}{J g} & 0 & 0 \\
0 & 0 & 0 & 0 & 1 \\
0 & 0 & 0 & -\omega^{2} & -2 \zeta \omega
\end{array}\right] \\
B=\left[\begin{array}{cc}
0 & 0 \\
0 & 0 \\
0 & \frac{-1}{J_{g}} \\
0 & 0 \\
\omega^{2} & 0
\end{array}\right], H=\left[\begin{array}{c}
\frac{\alpha}{J_{r}} \\
0 \\
0 \\
0 \\
0
\end{array}\right]
\end{gathered}
$$

where

$$
\gamma=\left.\frac{\partial M_{a}}{\partial \Omega_{r}}\right|_{x^{*}, u^{*}, d^{*}}, \alpha=\left.\frac{\partial M_{a}}{\partial v_{\mathrm{x}}}\right|_{x^{*}, u^{*}, d^{*}}, \beta=\left.\frac{\partial M_{a}}{\partial \theta}\right|_{x^{*}, u^{*}, d^{*}}
$$

We assume that only $\Omega_{r}, \Omega_{g}$ and $\theta$ are measurable. Therefore, the measurement output matrix $C_{y}$ will be

$$
C_{y}=\left[\begin{array}{lllll}
1 & 0 & 0 & 0 & 0 \\
0 & 0 & 1 & 0 & 0 \\
0 & 0 & 0 & 1 & 0
\end{array}\right]
$$

In Region 3 the generator torque $M_{g}$ is kept on its rated value and the blade pitch angle $\theta^{*}$ is obtained by solving

$$
M_{a}\left(\Omega_{\text {rated }}, v_{\mathrm{x}, 0}, \theta^{*}\right)=M_{\text {rated }}
$$

Thus the input matrix $B$ and controlled output matrix $C_{z}$ simplify to:

$$
B=\left[\begin{array}{lllll}
0 & 0 & 0 & 0 & \omega^{2}
\end{array}\right]^{T}, \quad C_{z}=\left[\begin{array}{lllll}
0 & 0 & 0 & 1 & 0
\end{array}\right]
$$

In Region 2, the blade pitch angle $\theta$ is maintained at zero, so (9) is not used, and we obtain a third order model with the state variables

$$
x=\left[\begin{array}{lll}
\Omega_{r} & \phi & \Omega_{g}
\end{array}\right]^{T} .
$$

The control input is the generator torque, $u(t)=M_{g}$. The linearized system 12 has matrices

$$
A=\left[\begin{array}{ccc}
\frac{\left(\gamma-C_{d}\right)}{J_{r}} & \frac{-K_{d}}{J_{r}} & \frac{C_{d}}{J_{r}} \\
1 & 0 & -1 \\
\frac{C_{d}}{J_{g}} & \frac{K_{d}}{J_{g}} & \frac{-C_{d}}{J_{g}}
\end{array}\right], B=\left[\begin{array}{c}
0 \\
0 \\
\frac{-1}{J_{g}}
\end{array}\right], H=\left[\begin{array}{c}
\frac{\alpha}{J_{r}} \\
0 \\
0
\end{array}\right]
$$


The measurement output matrix reduces to

$$
C_{y}=\left[\begin{array}{lll}
1 & 0 & 0 \\
0 & 0 & 1
\end{array}\right] .
$$

The rotor speed $\Omega_{r}$ is the controlled output, thus $C_{z}$ is

$$
C_{z}=\left[\begin{array}{lll}
1 & 0 & 0
\end{array}\right] \text {. }
$$

\section{Wind Turbine Control}

Here we introduce the control objectives and methodologies to be considered in our simulation studies. We discuss the specific performance objectives of turbine control, and articulate some measures for comparing the performance of different control methodologies. Lastly, we introduce the Exact Output Regulation control methodology that has been widely studied in the control systems literature for several decades. The principal novelty of our work lies in the application of this classical control methodology to a LIDAR-enhanced wind turbine.

\subsection{Wind Turbine Control Objectives}

Wind turbine control objectives may be divided into two categories: improving power production and reducing load fatigues. In Region 2 the power objective is to generate the maximum power from the available wind, while in Region 3, the objective is to maintain the power at the turbine's rated value. The performance metric for these power objectives are the mean and standard deviation of the associated signal. For example, the mean value for the generated power will be calculated with the following equation:

$$
P_{\text {mean }}=\frac{1}{T} \int_{0}^{T} P d t
$$

where $T$ is the time duration of measurement and $P$ is the generator output power measured by FAST. A smaller standard deviation of the rotor speed $\operatorname{std}\left(\Omega_{r}\right)$ indicates improved performance of the controller on retaining the rotor speed at the rated speed in Region 3. A smaller value for the standard deviation of the generated power $\operatorname{std}(P)$ indicates reduced frequency fluctuations of the power supplied to the power network; such fluctuations are known to be a problematic aspect of the injection of intermittent active power from large wind farms into the network [37,38]. In Region 2, a smaller value for the standard deviation of the tip speed ratio $\operatorname{st} d(\lambda)$ indicates the controller is more successful in maintaining the tip speed ratio at the optimal value for power generation.

Fatigue loads on the structure are caused by the oscillations induced by the wind and actuator variations. The standard metrics for measuring turbine fatigues are the Damage Equivalent Loads (DELs) which represent the damages caused by the structural loads accumulated during the lifetime of the turbine. The three principal loads considered in this paper are the torsional displacement on the drive train shaft, and the bending moments of the tower root and blade root. These points are under high strain and also compose the most expensive parts of the wind turbine. Therefore, load mitigation on these points are economically very desirable. Finally, for visualization in the frequency domain [24], power spectral density (PSD) of some of the measurements will be shown. PSD represents the spectral content of these signals. Normally, reduced high frequency spectral content is desirable as it implies reduced vibrations in the system components. 


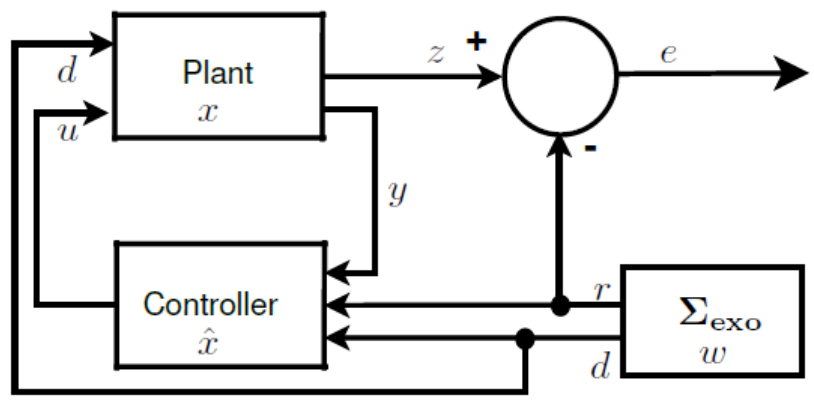

Figure 2: Block diagram of EOR control methodology

\subsection{The Exact Output Regulation Control Methodology}

EOR is a multi-variable LTI control methodology in which the plant is subject to known timevarying input disturbances that are to be rejected, and the plant outputs are required to track a known time-varying reference signal. The aim of EOR is to design a feedback control law which ensures that the plant dynamics are stable, and the output asymptotically converges to a desired reference signal while rejecting the disturbances. The following summary of EOR is taken from [39], which was adapted from [28].

The EOR control methodology considers a linear time-invariant multivariable system shown in the block diagram of Figure 2. The plant $\Sigma$ is assumed to be described by state equations in the form of (12), where $x, y$ and $z$ are respectively the plant state, measured output and regulated output. A known linear time-invariant exosystem $\Sigma_{\text {exo }}$ generates the autonomous time-varying reference signal $r$ and input disturbance signal $d$. The error signal $e=z-r$ represents the difference between the regulated output and the reference. The exosystem can be written in the form of:

$$
\Sigma_{\text {exo }}:\left\{\begin{aligned}
\dot{w}(t) & =S w(t), \quad w(0)=w_{0} \\
d(t) & =L_{d} w(t) \\
r(t) & =L_{r} w(t)
\end{aligned}\right.
$$

Here $S$ represents the exosystem dynamics and $w$ is the state of the exosystem. Output matrices $L_{d}$ and $L_{r}$ construct the disturbance and reference signals from the exosystem states. By defining

$$
\begin{aligned}
& E_{w}=H L_{d} \\
& D_{w}=-L_{r}
\end{aligned}
$$

we can replace $\Sigma$ in 12 with the error system $\Sigma_{e}$ :

$$
\Sigma_{e}:\left\{\begin{array}{l}
\dot{x}(t)=A x(t)+B u(t)+E_{w} w(t) \\
\dot{w}(t)=S w(t) \\
y(t)=C_{y} x(t) \\
e(t)=C_{z} x(t)+D_{w} w(t)
\end{array}\right.
$$

A feedback controller $u$ for the system $\Sigma_{e}$ is said to achieve exact output regulation [28] if the closed-loop system is internally stable and, for all initial states $x_{0}$ and $w_{0}$ of the plant and exosystem, the system satisfies $\lim _{t \rightarrow \infty} e(t)=0$. Ensuring the error signal vanishes means that the input disturbance is asymptotically rejected, and the controlled output $z$ asymptotically 
tracks the desired reference signal $r$. For the case where all states are measurable, we have $y=x$ and state feedback can be used to achieve exact output regulation according to the following theorem:

Theorem 3.1 [28] Assume system $\Sigma_{e}$ in 22] satisfies the following assumptions

(A.1) The pair $(A, B)$ is stabilizable.

(A.2) The matrix $S$ is anti-Hurwitz-stable.

(A.3) There exist matrices $\Gamma$ and $\Pi$ satisfying

$$
\begin{aligned}
\Pi S & =A \Pi+B \Gamma+E_{w} \\
0 & =C_{z} \Pi+D_{w} .
\end{aligned}
$$

Let $F$ be any matrix such that $A+B F$ is Hurwitz stable, and let $G=\Gamma-F \Pi$. Then the state feedback control law

$$
u=F x+G w,
$$

achieves exact output regulation for $\Sigma_{e}$.

The Sylvester matrix equations (23)-(24) are known as the regulator equations and generic solvability conditions are given in [28]. The matrix $S$ is anti-Hurwitz stable if none of its eigenvalues are stable. In fact, this assumption is not essential and was adopted in [28] only to avoid a trivial problem formulation in which output regulation is achieved by default because the exosystem states vanish.

In practice, it is not always possible to measure all states of the plant, and an estimate $\hat{x}$ of the plant state must be constructed using the measured output $y$. The following theorem gives conditions under which exact output regulation may be achieved with a dynamic measurement feedback controller.

Theorem 3.2 [28] Assume the system $\Sigma_{e}$ in (22) satisfies the assumptions (A.1)-(A.3). Further, assume the matrix pair

$$
\left(\left[\begin{array}{ll}
C_{y} & 0
\end{array}\right],\left[\begin{array}{cc}
A & E_{w} \\
0 & S
\end{array}\right]\right)
$$

is detectable. Then the exact output regulation problem is solvable by a dynamic measurement feedback controller of the form

$$
\Sigma_{c}:\left\{\begin{aligned}
{\left[\begin{array}{c}
\dot{\hat{x}}(t) \\
\dot{w}(t)
\end{array}\right] } & =\left[\begin{array}{cc}
A & E_{w} \\
0 & S
\end{array}\right]\left[\begin{array}{c}
\hat{x}(t) \\
w(t)
\end{array}\right]+\left[\begin{array}{c}
B \\
0
\end{array}\right] u(t) \\
& +\left[\begin{array}{c}
K_{A} \\
0
\end{array}\right]\left(\left[\begin{array}{ll}
C_{y} & 0
\end{array}\right]\left[\begin{array}{c}
\hat{x}(t) \\
w(t)
\end{array}\right]-y(t)\right) \\
u(t) & =F \hat{x}(t)+G w(t)
\end{aligned}\right.
$$

where $F$ and $K_{A}$ are such that $A+B F$ and $A+K_{A} C_{y}$ are both Hurwitz stable matrices, and $G=\Gamma-F \Pi$.

In sections 4 and 5, we discuss the application of EOR to a LIDAR-enhanced wind turbine. For a given mean wind speed $v_{\mathrm{x}, 0}$, we first develop a state model $\Sigma$ as in (12), with coordinates homogenised to the mean wind speed. The appropriate turbine model and controller are used for Region 2 or 3 according to whether the mean wind speed is below or above the rated wind 
speed of $11.4 \mathrm{~m} / \mathrm{s}$. We then include wind dynamics to obtain the error system $\Sigma_{e}$ as in (22), also in homogenised coordinates.

The vector $w$ represents the deviations of the perpendicular wind speed (as measured by LIDAR) from its mean value, and its dynamics are modelled by the matrix $S$. The wind speed deviation is modeled as an input disturbance $\bar{d}$, and effective disturbance rejection means that the effect of $\bar{d}$ on the turbine response is attenuated. The wind speed deviation also determines the reference signal $\bar{r}$. In Region 2 , the reference is the rotor speed that delivers the optimal tip speed ratio. In Region 3, the reference is the blade pitch angle that will maintain the rotor speed at the turbine's rated power.

Theorems 3.1 and 3.2 are applicable to the nominal linear system $\Sigma_{e}$, and hence the application of the EOR control law (26) to the nonlinear turbine dynamics (as modelled by FAST) cannot be expected to achieve exact output regulation. However, the simulation results to be presented in Section 6 will show that the EOR control method achieves the required rotor speeds for optimal energy harvesting with smoother control actuation. This leads to reduced fatigue loads, relative to alternative control methods that do not incorporate wind dynamics into their controller design.

\subsection{Alternative Turbine Control Methodologies}

To demonstrate the effectiveness of the EOR method in reducing fatigue loads, in Section 6 we shall compare its performance with two alternative control methods whose application to turbines have been widely studied. Here, we briefly describe these alternative methods.

One of the most well-known wind turbine control methods is DAC, which uses wind estimation data to reduce or cancel persistent input disturbances. Similar to EOR, DAC assumes a linear plant model of the form (12). Disturbance states are created by the augmentation of a state-estimator in a state feedback controller by an assumed-waveform model. These disturbance states are used to reduce or counteract the persistent disturbance effects of the wind. The wave-form model is commonly assumed to take a constant value ( $20,40,41])$ in the form

$$
\begin{aligned}
\dot{z}_{d}(t) & =0 \\
d(t) & =z_{d}(t),
\end{aligned}
$$

where $z_{d}(t)$ is the state of the disturbance model. In this paper, the LIDAR-enhanced variant of DAC known as DAC+LIDAR [4] will be used for comparisons, in which $d(t)$ is taken as the LIDAR longitudinal wind measurement. Thus in our performance comparisons in Section VI, EOR and DAC+LIDAR have access to the same wind preview information. For simplicity, in the following we shall use DAC when referring to the LIDAR-enhanced version. The DAC control law is

$$
u(t)=F x(t)+G_{d} z_{d}(t),
$$

where $F$ is a state feedback matrix chosen to place the closed-loop poles at certain desired locations, and $x$ is the state variable of the wind turbine model. Then, if possible, the wind disturbance state gain $G_{d}$ is chosen to exactly cancel out the wind disturbance by solving

$$
B G_{d}+H=0
$$

for $G_{d}$ in which $B$ and $H$ are defined in 12 .

When (30) is solvable, the DAC control methodology may be viewed as a special case of the EOR control methodology. If we apply the simplifying assumptions $S=0$ and $L_{d}=1$ to 
(21), we obtain $E_{w}=H$ and

$$
\begin{aligned}
\dot{w}(t) & =0 \\
d(t) & =z_{d}(t)
\end{aligned}
$$

which are identical to (27)-(28) with $w=z_{d}$. Using $\Pi=0$ in the EOR control law gives $\Gamma=G_{d}$, so the first regulator equation 23 becomes

$$
0=B G_{d}+H
$$

which is 30 .

The equation (30) may not be solvable if the vector $B$ has zero elements. Then $G_{d}$ is chosen by the minimization problem

$$
\operatorname{argmin}_{G_{d}}\left\|B G_{d}+H\right\|_{2} .
$$

In such cases, DAC will not be able to exactly cancel the disturbances.

We note a number of similarities and differences between EOR and DAC. Both use a state feedback law to stabilise the closed-loop dynamics and a feedforward term to cancel input disturbances. Where DAC models the disturbance as a constant, the dynamic exosystem used in EOR enables greater flexibility in the modelling of the disturbance. Moreover, under mild system assumptions of controllability and observability, (23)-(24) are solvable. By contrast, only approximate solutions can be obtained for (30), leading to only approximate disturbance cancellation. Additionally, DAC cannot ensure the output tracks any desired time-varying reference.

Hence we can expect better disturbance rejection performance from EOR as it has access to derivatives of the disturbance input. Conversely, DAC only has access to the absolute value of the disturbance. Moreover, non-solvability of the DAC minimization equation (34) can occur if the disturbance input vector $H$ and control input vector $B$ are orthogonal. In such cases, obtaining a non-zero solution for $G_{d}$ will require some plant model reduction, leading to reduced control performance.

In our simulation results, we shall also compare the control performance of EOR and DAC against a Baseline method of wind turbine control, employing a proportional torque controller of the kind commonly used in the industry. The standard (Baseline) controller for Region 2 is a generator torque reference proportional to the square of the rotational speed of the rotor

$$
M_{g}=k \Omega_{r}^{2}
$$

where $k$ is given by

$$
k=\frac{1}{2} \rho \pi R^{5} \frac{C_{p}\left(\lambda^{*}, 0\right)}{\left(\lambda^{*}\right)^{3}} .
$$

In Region 3, the Baseline controller is a PI regulator which is set to eliminate rotor speed error by generating the required references for the blade pitch angle. The feedback information is taken from the rotor speed $\Omega_{r}$ and compared against the rated rotor speed. The rotor speed error is then fed into a conventional PI controller to generate the pitch command $\theta_{c}$ in the following form

$$
\begin{aligned}
\Delta \Omega & =\Omega_{r}-\Omega_{0} \\
\theta_{c} & =K_{p} \Delta \Omega+K_{i} \int \Delta \Omega d t
\end{aligned}
$$

The design procedure for determining the proportional and integral gains $K_{p}$ and $K_{i}$ is described in 40$]$. 


\section{Synthesizing Disturbance and Reference Exosystems for Wind Turbines}

A key component of the EOR control methodology introduced in Section 3.2 is a linear exosystem in (21) that generates the known disturbance and reference signals. In this section, we describe how LIDAR measurement data can be used to synthesize linear exosystem dynamics to represent short-term wind evolution with a high fidelity.

If $f$ is the focal distance of the CW-LIDAR and $v_{\mathrm{x}, 0}$ is the mean longitudinal wind speed, a time window of $T_{f}=\frac{f}{v_{x, 0}}$ of wind preview information will be available. Since $T_{f}$ depends on the mean wind speed, a constant preview length of $0<T_{p l}<T_{f}$ is assumed to cover the whole range. In this work, we assume $f=60 \mathrm{~m}$ and our largest wind speed is $24 \mathrm{~m} / \mathrm{s}$, hence $T_{p l}=1.5 s$ is suitable. To find an exosystem that can accurately model the longitudinal wind signal $v_{\mathrm{x}}$, an auto-regressive model is fitted to the longitudinal wind speed signal $\hat{v}_{\mathrm{x}}$ provided by the LIDAR (our LIDAR model will be discussed in Section 5.0.2) over the time window $\left[0, T_{p l}\right]$. Therefore, the combined exosystem and turbine model $(12)$ are constructed in discrete time. The exosystem dynamics are modeled with an LTI difference equation

$$
w_{\mathbf{x}}[n]=a_{1} w_{\mathbf{x}}[n-1]+a_{2} w_{\mathbf{x}}[n-2]+\cdots+a_{N} w_{\mathbf{x}}[n-N],
$$

in which

$$
w_{\mathbf{x}}[n]=\hat{v}_{\mathbf{x}}[n]-\hat{v}_{\mathbf{x}, 0}
$$

is the deviation of the LIDAR-measured longitudinal wind speed $\hat{v}_{x}$ from the mean LIDAR longitudinal wind speed $\hat{v}_{\mathbf{x}, 0}$, at sampling instant $n$. The $a_{k}$ are the system coefficients and $N$ is the order of exosystem dynamics to be chosen.

Choosing higher values of $N$ yields better representation of the wind deviation, leading to better disturbance rejection and reference tracking. However, aggressive disturbance rejection and reference tracking are contradictory to fatigue load reduction. This is because perfect disturbance rejection would require all the turbine states to precisely track their equilibrium value against the wind speed, which would increase tower deflection and drive-train torsion. Thus, a trade-off needs to be made between higher fidelity modeling of the wind (using larger $N$ values) and reducing the time-variation of the control input signal (using lower $N$ values).

Throughout this work, $N=2$ has been empirically chosen to generate exosystems that are computable in real time. Hence we introduce a state vector for the exosystem as $w[n]=$ $\left[w_{\mathbf{x}}[n], w_{\mathbf{x}}[n-1], w_{\mathbf{x}}[n-2]\right]^{T}$, and the exosystem dynamics are given by

$$
w[n+1]=\underbrace{\left[\begin{array}{ccc}
a_{1}^{*} & a_{2}^{*} & a_{3}^{*} \\
1 & 0 & 0 \\
0 & 1 & 0
\end{array}\right]}_{S} w[n],
$$

The optimal coefficients $a_{k}^{*}$, for $1 \leq k \leq 3$ may be obtained by passing a batch of sampled LIDAR measurements into a Least Square Algorithm every $T_{p l}$ seconds. However, a batch least square method has to perform large matrix inversions at every $G$ matrix update event which might be not feasible for real-time applications. By using Recursive Least Square (RLS) instead, this computation load is distributed evenly for each simulation step. The RLS estimator for $a_{k}^{*}$ coefficients are shown in Figure 3 . Thus the $a_{k}$ coefficinets, and hence the $S$ matrix, are updated at each time step, with frequency $10 \mathrm{~Hz}$. With a forgetting factor of 0.90 in the RLS, the weight of each sampled data becomes almost zero after 6 seconds, as $0.90^{60} \approx 0.001$. The 


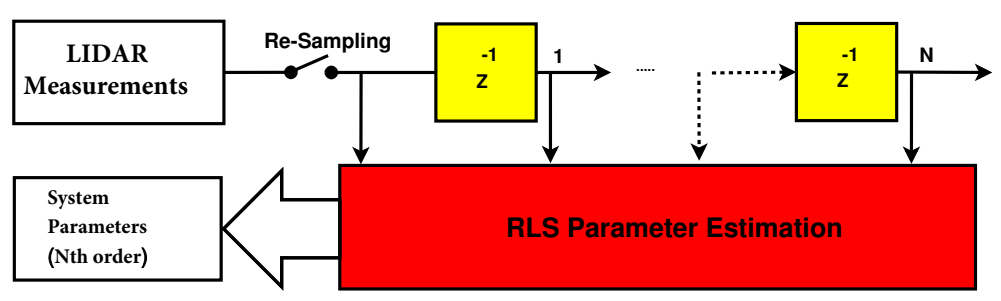

Figure 3: Recursive least square estimation of parameters

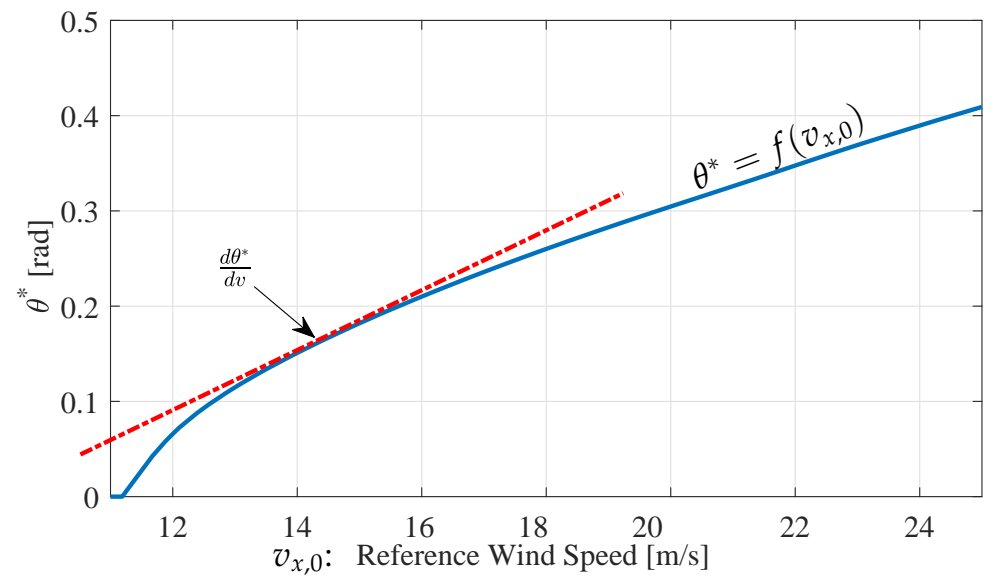

Figure 4: Equilibrium locus of the optimal blade pitch angle $\theta^{*}$ for Region 3 wind speeds (Blue) and its tangent (Red)

regulator equations (23)-24), and consequently the feed-forward gain matrix $G$, are updated every 6 seconds.

To complete the exosystem model $\Sigma_{\text {exo }}$ in $(21)$, output matrices $L_{d}$ and $L_{r}$ should be also determined. The disturbance input is the deviation of the longitudinal wind speed from the mean value $\bar{d}(t)=\hat{v}_{\mathbf{x}}(t)-\hat{v}_{\mathbf{x}, 0}(t)=w_{\mathbf{x}}(t)$, so

$$
L_{d}=\left[\begin{array}{lll}
1 & 0 & 0
\end{array}\right]
$$

As discussed in Section 2.2, in Region 2 the controlled output is the rotor speed $\Omega_{r}^{*}$, and from (2) we have, in homogenised coordinates, $\bar{\Omega}_{r}=\frac{\lambda^{*} w_{\mathrm{x}}}{R}$. Taking $\bar{\Omega}_{r}(t)$ as the time-varying reference signal to be tracked by the EOR controller, we need

$$
L_{r}=\left[\begin{array}{lll}
\lambda^{*} & 0 & 0
\end{array}\right]
$$

For a Region 3 mean longitudinal wind speed $v_{\mathrm{x}, 0}$, the controlled output is the blade pitch angle $\theta^{*}$, obtained by solving (16). Figure 4 shows the graph of $\theta^{*}$ as a function of $v_{\mathrm{x}, 0}$.

To obtain the time-varying reference signal $\bar{\theta}(t)$ in homogenised coordinates, we use the first order approximation

$$
\bar{\theta}=\frac{d \theta^{*}}{d v_{\mathrm{x}, 0}} w_{\mathrm{x}}
$$

Thus for Region 3,

$$
L_{r}=\left[\begin{array}{lll}
\frac{d \theta^{*}}{d v_{\mathrm{x}, 0}} & 0 & 0
\end{array}\right]
$$




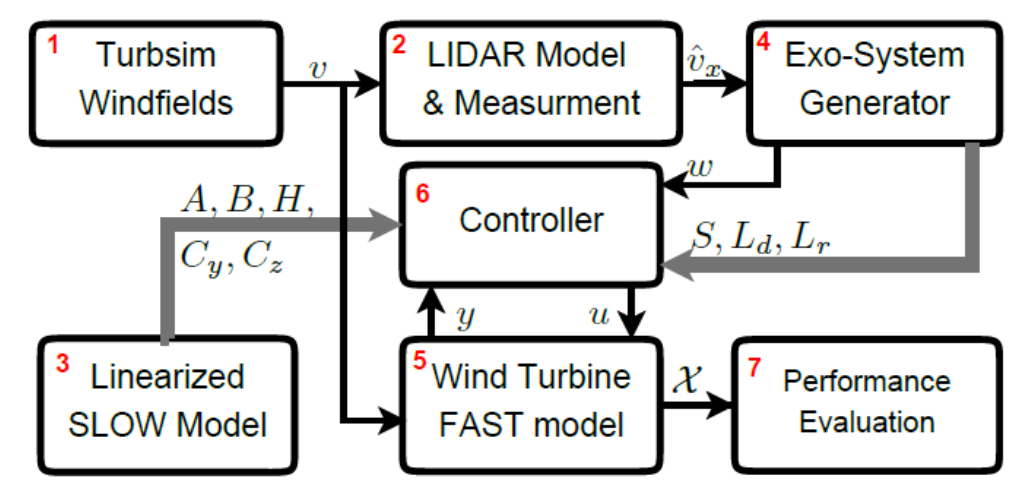

Figure 5: Block Diagram of TOR. Thick gray lines represent matrices and thin black lines represent signals.

\section{Turbine Simulation Environment}

In this section, we describe our turbine simulation environment, developed in Simulink ${ }^{\circledR}$, and named the Turbine Output Regulator (TOR). Its purpose is the simulation and comparison of the control performance of the EOR, DAC and Baseline control methodologies for the NREL $5 M W$ FAST turbine model. The block diagram of TOR is illustrated in Figure 5 , It is comprised of seven subsystems as follows:

1. The TurbSim package [33] for the simulation of realistic wind fields.

2. The LIDAR simulator based on [42].

3. The linearized model of the nonlinear Simplified Low-Order of Wind (SLOW) Turbine model.

4. The linear exosystem generator obtained from LIDAR data, as described in Section 4 .

5. The high fidelity wind turbine simulator FAST.

6. The controller subsystem implements the Baseline, DAC and EOR controllers described in Section 3 .

7. Performance measurement code to calculate metrics related to the power generation and DELs.

We now briefly describe each subsystem:

\subsubsection{TurbSim Wind Field Simulator}

TurbSim is a full-field, turbulent-wind simulator developed by NREL using stochastic models to generate realistic three-dimensional wind field vectors $v$, with components for the longitudinal, crosswise and vertical components of the wind, in arbitrary resolution. The detailed parameters of wind input files are the vertical stability parameter $R i_{T L}$, shear exponents $\alpha_{D}$ and the mean friction velocity $u_{D}^{\star}[43]$ which are set on the default values in TurbSim to generate 1 hour of wind information. Figure 6 shows a TurbSim-generated Class-A intensity wind signal of one hours duration, with a mean longitudinal wind speed of $v_{\mathbf{x}, 0}=18 \mathrm{~m} / \mathrm{s}$. We also show the 


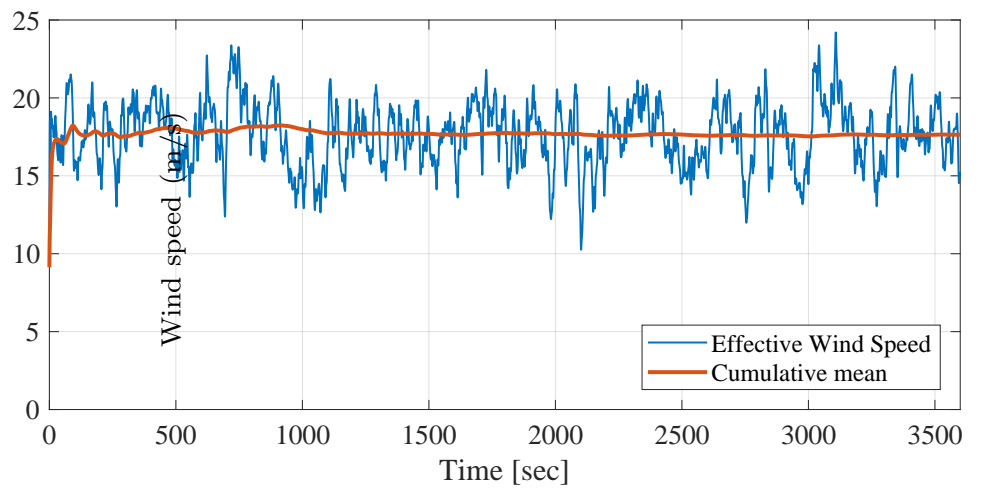

Figure 6: Effective Hub-height wind speed of Class-A TurbSim wind signal and its cumulative mean.

cumulative mean of the signal. The output $v$ from TurbSim in Figure 5 is the wind field vector which is applied to both the FAST turbine simulator and the LIDAR simulator.

The $5 M W$ reference wind turbine is a class-A wind turbine, and consequently, the FAST simulator is exposed to a broad range of Class-A intensity wind fields generated by TurbSim according to IEC-61400-1 standard [44]. These wind fields have mean longitudinal wind speeds from 8 to $24 \mathrm{~m} / \mathrm{s}$ with resolution steps of $2 \mathrm{~m} / \mathrm{s}$. Wind speeds below $8 \mathrm{~m} / \mathrm{s}$ are not considered, as 31 does not recommend the use of a Baseline torque controller of the form (35) below this wind speed.

\subsubsection{LIDAR Simulator}

We use the continuous wave CW-LIDAR model described in [42] to simulate the longitudinal wind speed $v_{\mathrm{x}}$ at a specific distance from the turbine blades by focusing the laser beam at that location. Figure 7 depicts the coordinate system and geometrics of the LIDAR placement on the wind turbine nacelle.

Twenty-four evenly distributed measuring points on a circular cross-section of the wind vector $v$ at focal distance $f$ from the rotor plane are scanned by the LIDAR beam, and spatial averaging is applied to the wind speeds along the length of each measurement beam [42]. The effect of the averaging is equivalent to passing the wind signal through a non-phase distorting low-pass filter whose $3 d B$ bandwidth is determined by

$$
B W_{3 d B}=\frac{87}{f^{2}} .
$$

The constant 87 is based on specific parameters of the LIDAR used in [42]. Averaging Riemannian sums of the 24 measurements in the cross-section yields $\hat{v}_{\mathrm{x}}$, an approximation to the longitudinal speed $v_{\mathrm{x}}$. In Figure 8 the overlay comparison between the real hub-height wind signal and the simulated LIDAR output $\hat{v}_{\mathrm{x}}$ with a focal distance of 60 metres is shown.

TurbSim generates wind signals according to Taylor's Frozen Wind Hypothesis, which models the wind field as a turbulence box moving towards the wind turbine at its average wind speed. Thus the wind field is assumed not to evolve between the LIDAR focal point and the blades. This hypothesis is appropriate for relatively flat terrain where geological features do not interact with the air flow between the measurement point and blades. Additionally, the induction zone caused by the turbine itself certainly causes some wind evolution between the LIDAR focal point and the blades. 


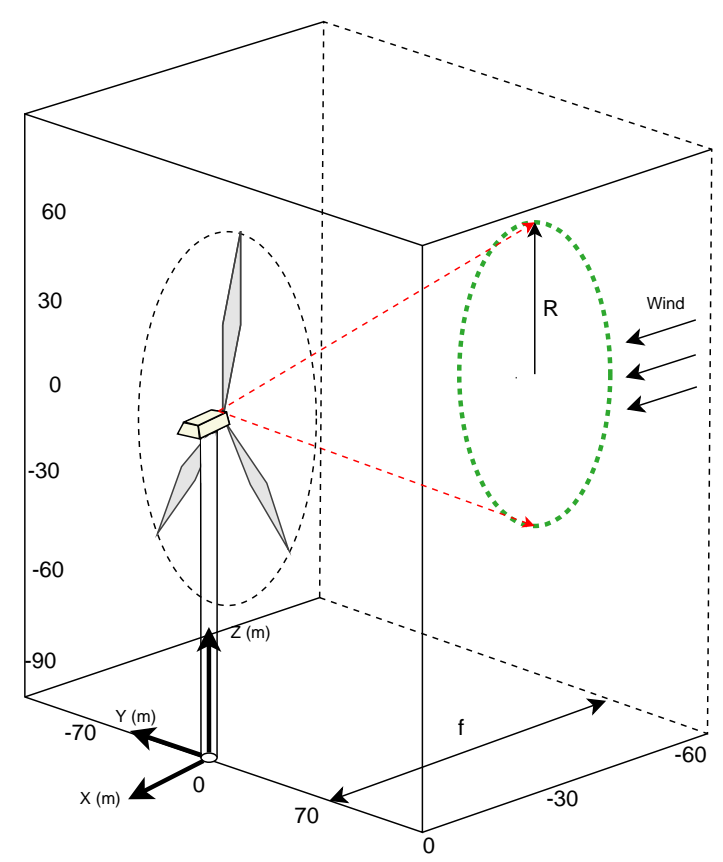

Figure 7: Coordinate system of wind turbine and nacelle mounted LIDAR shown by $\mathrm{x}, \mathrm{y}$ and z. The distance of the focal point of CW-LIDAR is shown by $f$ and $R$ is the scan radius.

\subsubsection{Linearized low order model}

In order to compute a low-order linearized turbine model, knowledge of the mean wind speed $v_{\mathrm{x}, 0}$ is required. It is apparent from Figure 6 that after some 100 seconds, the cumulative mean of the longitudinal wind signal $v_{\mathrm{x}}$ gives a good approximation to the mean longitudinal wind speed $v_{\mathrm{x}, 0}$, and hence we use the cumulative mean, denoted by $\hat{v}_{\mathrm{x}, 0}$, as the linearization point satisfying (10). The linearized model (12) is obtained as described in Section 2.4, based on the parameters given in Table 1 in coordinates homogenised to the assumed mean wind speed. This linear model is passed to the controller subsystem for the computation of the DAC and EOR control inputs.

\subsubsection{Wind Exosystem Generator}

This subsystem uses the cumulative mean wind speed to construct the homogenised wind signal $w_{\mathrm{x}}$ in (40). Matrices $S, L_{r}$ and $L_{d}$ for the exosystem $\Sigma_{\text {exo }}$ (21), are obtained as in Section 4. The exosystem state variable $w$, disturbance $d$ and reference $r$ are passed to the controller subsystem.

\subsubsection{FAST Turbine Simulator}

To simulate the response of the $5 M W$ HAWT turbine, we use the NREL FAST 7 code. A compiled MATLAB ${ }^{\circledR}$ S-Function of the FAST code is used to link the designed controllers to FAST in Simulink ${ }^{\circledR}$. Table 2 lists the degrees of freedom applicable to the on-shore $5 \mathrm{MW}$ wind turbine that have been activated in FAST for our simulation. FAST does not include a model for pitch actuator, so a second order servo-system according to Table 1 has been added to the Simulink ${ }^{\circledR}$ environment.

The FAST model receives the wind field vector $v$ from TurbSim and uses it to compute the turbine state vector $\bar{x}$ and measured output signal $\bar{y}$. These signals are passed to $\Sigma_{c}$ in (26) for 


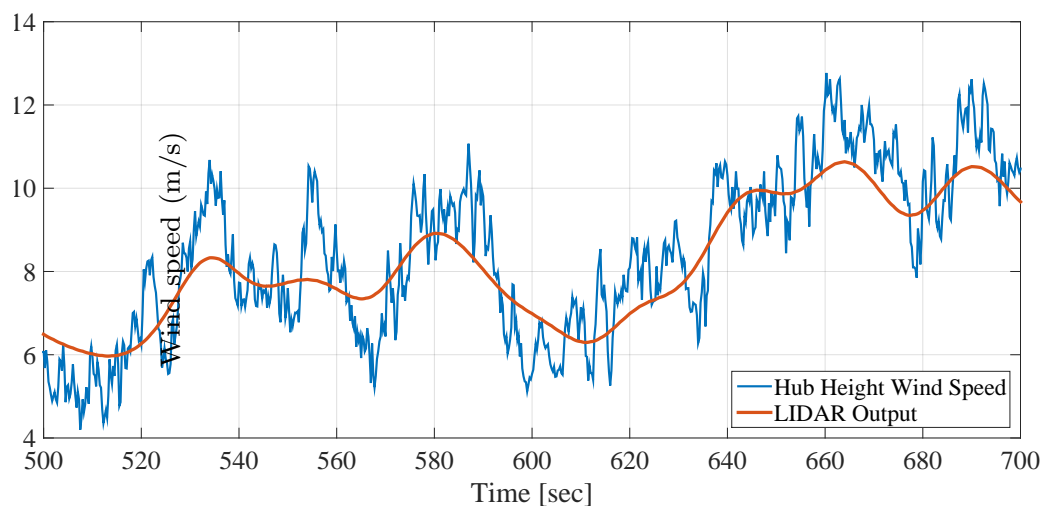

Figure 8: Blue: A 200 Seconds sample of a Region 2 Class-A wind with mean speed of $8 \mathrm{~m} / \mathrm{s}$, measured at the hub height. Red: Output of the CW-LIDAR model.

Table 2: Enabled DOFs in FAST code

\begin{tabular}{lcr}
\hline \hline Enabled mode & No. of DOFs & Total \\
\hline Generator & 1 & 1 \\
Drive-train Torsion & 1 & 1 \\
$1^{\text {st } \& \quad 2^{\text {nd }} \text { fore-aft }}$ & 1 & 2 \\
tower bending & & \\
$1^{\text {st } \& \quad 2^{\text {nd }} \text { side-side }}$ & 1 & 2 \\
tower bending & & \\
$1^{\text {st }}$ edge-wise blade & $1 \times 3$ & 3 \\
$2^{\text {nd }}$ edge-wise blade & $1 \times 3$ & 3 \\
$1^{\text {st }}$ flap-wise blade & $1 \times 3$ & 3 \\
$2^{\text {nd }}$ flap-wise blade & $1 \times 3$ & 3 \\
\hline Total DOFs & & 18 \\
\hline
\end{tabular}

computation of the control input $\bar{u}$. Finally, all measurements contained in the FAST output $\log$ files, represented by $\mathcal{X}$ in Figure 5, are passed to the performance measurement subsystem. These include the blade root and tower root bending moments, rotor and generator speeds, and torsion of the drive shaft, as well as the pitch angle and generator torque input commands.

\subsubsection{Controller subsystem}

This subsystem computes the EOR, DAC and Baseline controller input signals $u$, and passes them to the FAST simulator. The EOR dynamic measurement feedback controller $\Sigma_{c}$ is given by (26). The state feedback matrix $F$ is chosen by the LQR algorithm with $Q=C_{z}^{T} C_{z}$, and $R$ is empirically chosen to avoid control input saturation. The same matrix $F$ is used for the DAC controller. The estimated homogenised state vector $\hat{\bar{x}}$ is obtained using the homogenised measured outputs $\bar{y}$ received from the FAST simulator. The observer feedback gain matrix $K_{A}$ is determined by a Kalman filter. The wind turbine model in 12 does not have a process noise term, and the output measurements obtained from the outputs of the FAST contain only numerical errors, so the process noise covariance matrix of the Kalman filter can be neglected, and a very small measurement noise covariance matrix is sufficient. 


\subsubsection{Performance Measurement}

The damage equivalent loads computed are the tower root fore-aft bending moment $M_{\mathrm{y} T}$, tower root side-to-side bending moment $M_{\mathrm{x} T}$, blade root flapwise bending moment $M_{\mathrm{y} B}$, blade root edgewise bending moment $M_{\mathrm{x} B}$, and $L S S$ torque. These are computed using the RainFlow-Counting method [45], with DEL computations performed with the Rain-Flow-CountingAlgorithm open source MATLAB ${ }^{\circledR}$ code [34]. This subsystem also computes the average power generated $P_{\text {mean }}$, and for Region 3 operation we compute the power standard deviation $\operatorname{std}(P)$ and rotor speed standard deviation $\operatorname{std}\left(\Omega_{r}\right)$. Smaller power standard deviation indicates the power generation is maintained close to the rated value of $5 M W$. For Region 2 operation we compute the tip speed ratio standard deviation $\operatorname{std}(\lambda)$, with smaller values indicating better tracking of the optimal tip speed ratio $\lambda^{*}$.

Additionally, the measurement subsystem provides spectral analysis of the tower fore-aft bending moment, tower side-to-side bending moment, blade flap-wise bending moment, low speed shaft torque, pitch rate and the generated power. Reduced high frequency content in the power spectral density (PSD) of these variables implies reduced fluctuations of the measured variable. For bending moment (tower or blade) signals, the integral of the amplitude of the PSD over the frequency range is an indicator of the wind energy dissipated within the turbine tower or blade. The consequence of this energy dissipation is fatigue accumulation in the tower or blade, and thus reduced PSD amplitudes are associated with lower lifetime turbine damage.

The TOR environment is able to compute one hour of turbine response simulation in 20 minutes of CPU time on a contemporary desktop PC, and hence the output regulation control methodology can be expected to be suitable for real-time realization on a wind turbine.

\section{$6 \quad$ Results and Comparisons}

We now present the simulation results from our investigation of the performance of the EOR, DAC and Baseline controllers introduced in Section 3. The simulations use class-A turbulent wind signals with mean wind speeds ranging between $8 \mathrm{~m} / \mathrm{s}$ and $24 \mathrm{~m} / \mathrm{s}$, as described in Section 5.0.1. The first 100 seconds of turbine response are excluded from the comparisons, as this time is required for the initialization of the DAC and EOR controllers.

Fatigue load DELs, standard deviation of rotor speed and generated power are shown for a range of wind speeds in both operating regions. A colour convention is used to represent the results for different controllers throughout this section as follows: Green represents outputs from a Baseline controller, Blue represents the DAC and EOR is shown by Red.

Figures 9 and 10 provide some illustrative time-domain comparisons. They show 800 seconds of turbine response data under the three controllers for class-A turbulent wind signals of mean speeds 8 and $18 \mathrm{~m} / \mathrm{s}$. The responses from the EOR controller exhibit smaller fluctuations than the two alternative controllers, particularly in the LSS torque variations. The control input graphs for the generator torque (Region 2) and blade pitch rate (Region 3) reveal that EOR exerts considerably smoother control actuation than both Baseline and DAC.

\subsection{Damage Equivalent Load Controller Performance Comparisons}

Figures 11a to 11d show fatigue loads for mean wind speeds ranging between $8 \mathrm{~m} / \mathrm{s}$ to 24 $\mathrm{m} / \mathrm{s}$. Results for Region 2 are distinguished with a gray background. In figure 11a, tower root fore-aft bending moment shows that DAC and EOR both improve considerably over exo in 

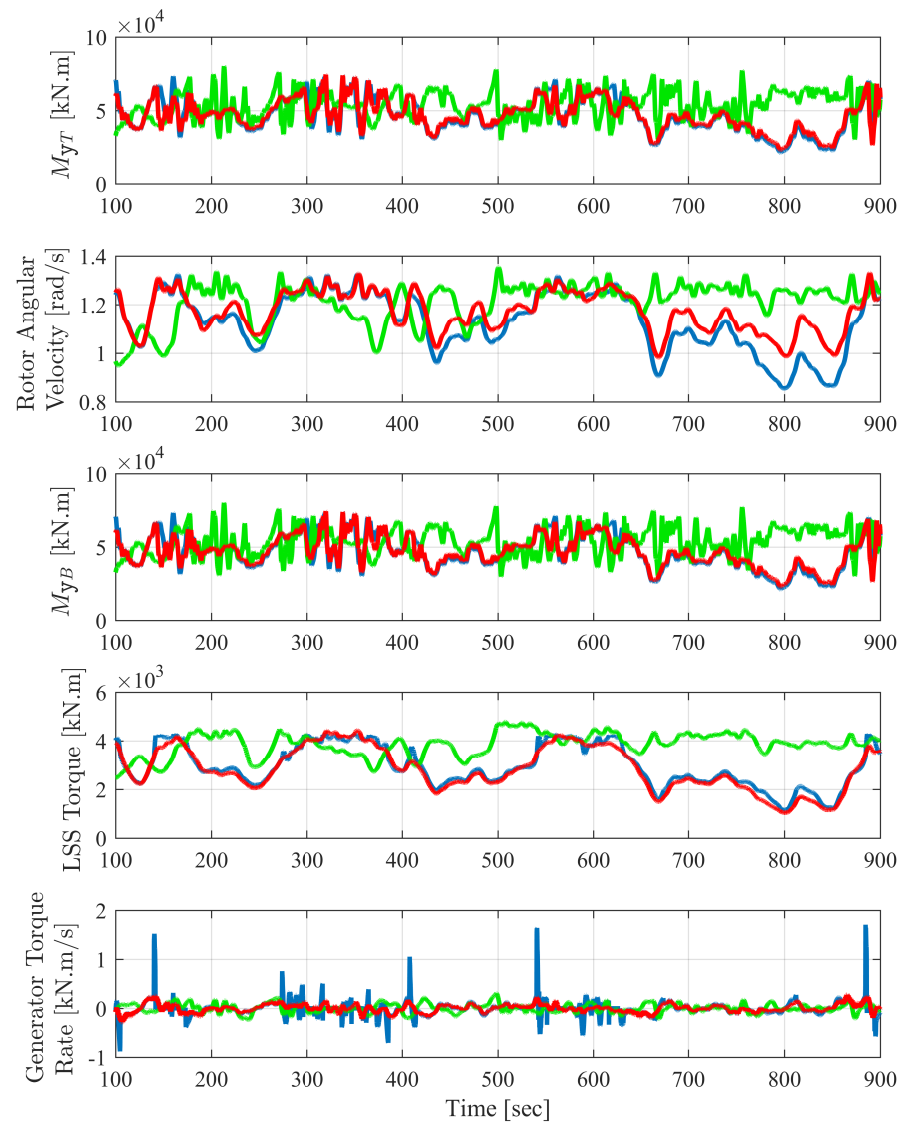

Figure 9: Illustrative responses for mean wind speed of $9 \mathrm{~m} / \mathrm{s}$ using Baseline control (Green), DAC (Blue) and EOR (Red).

reducing this load. For tower root side-to-side bending moment, Figure $11 \mathrm{~b}$ does not indicate a consistent improvement for EOR or DAC over Baseline, apart from the transition region near $12 \mathrm{~m} / \mathrm{s}$ where EOR improved greatly over both Baseline and DAC. In Figure 11c, low speed shaft torque performance is illustrated, showing EOR outperforms both alternative controllers across all wind speeds. For blade root flap-wise moments (Figure 11d), EOR and DAC were consistently better than Baseline in reducing the blade loads.

In order to compare lifetime DELs under each controller, the DELs applicable at each mean wind speed must be averaged across the operating range, with weighting according to the relative frequency of each mean wind speed. Figure 12 represents a sample Weibull distribution of wind speed variation from measured data at the height of $102 \mathrm{~m}$ in Bremerhaven, Germany, recorded during the winter of 2009 [46]. We have used this distribution to weight the performance results shown in Figure 11a to 11d, and the calculated lifetime values are shown in the first three rows of Table 3. The last two rows of this table show the percentage of improvements of EOR and DAC against the Baseline controller. Positive numbers indicate improvement relative to Baseline, while negative values indicate inferior performance.

Table 3 illustrates that, without reducing power generation, both the EOR and DAC controllers have been able to reduce lifetime DEL loads, in comparison with Baseline. However, the DAC performance showed deterioration in LSS torque and tower root side-to-side bending 

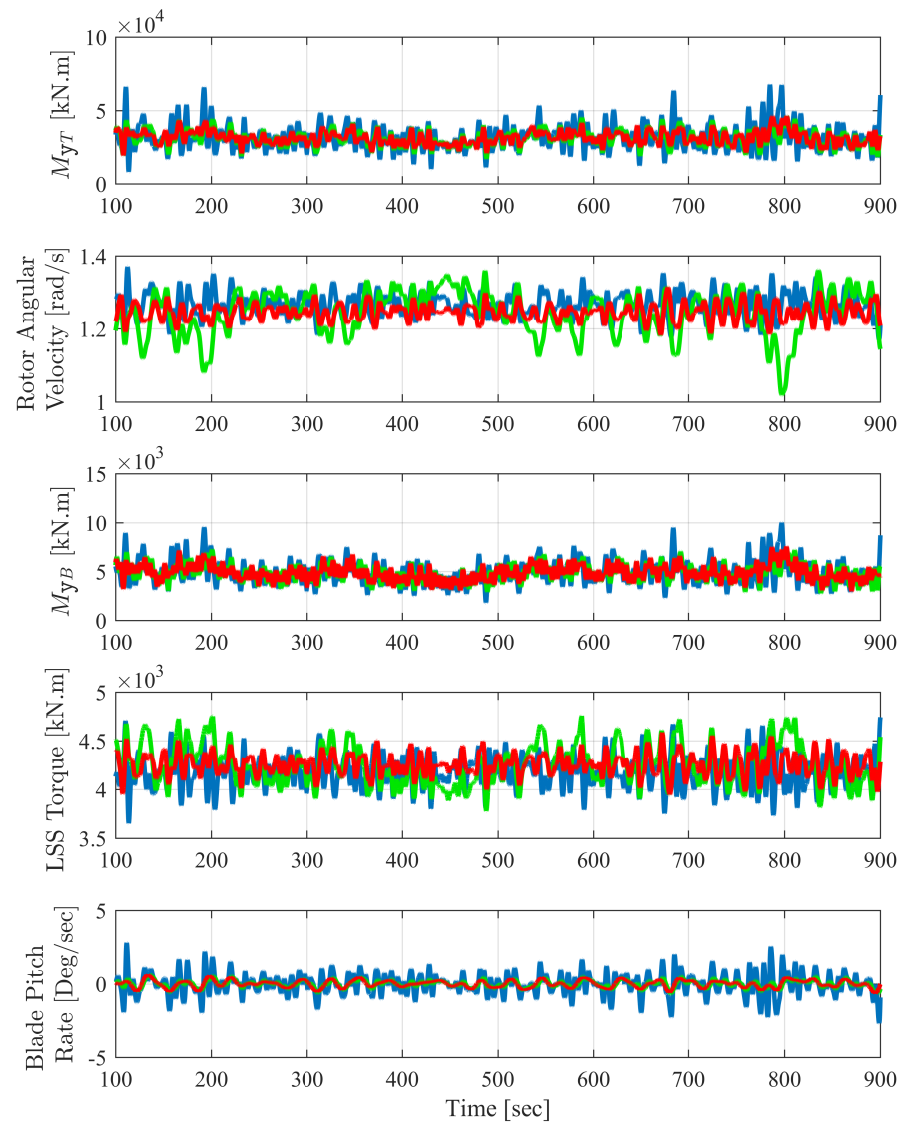

Figure 10: Illustrative responses for mean wind speed of $18 \mathrm{~m} / \mathrm{s}$ using Baseline control (Green), DAC (Blue) and EOR (Red).

moment, relative to the Baseline controller. By contrast, with the exception of $M_{\mathrm{x} B}$, EOR has been able to improve on Baseline for all the DEL metrics by margins of between $13 \%$ and $41 \%$.

Figure 14 shows power spectral densities for $M_{\mathrm{y} T}, M_{\mathrm{x} T}, L S S$ and $M_{\mathrm{y} B}$ for a mean wind speed of $20 \mathrm{~m} / \mathrm{s}$. In very low frequencies all controllers show similar spectral content, but above $0.02 \mathrm{~Hz}$, EOR has superior attenuation. As can be seen in Figure 13a for the tower root fore-aft bending moment $M_{\mathrm{y} T}$, EOR shows the most reduction around $0.05 \mathrm{~Hz}$ while for higher frequencies the EOR performance is comparable to DAC. For the tower root side-to-side bending moment $M_{\mathrm{x} T}$ shown in Figure $13 \mathrm{~b}$, similar improvements can be seen for EOR. The excitation around $0.32 \mathrm{~Hz}$ represents the first tower root side-to-side natural frequency [31]. Since the tower natural frequency is not considered in the reduced model (7)-(9), this frequency is not attenuated by any of the controllers. For low speed shaft torque in Figure 13c, DAC falls short of the other controllers below $0.05 \mathrm{~Hz}$ while EOR maintains better attenuation across the spectrum.

Blade root flap-wise moments $M_{\mathrm{y} B}$ in Figure $13 \mathrm{~d}$ show a peak at $0.2 \mathrm{~Hz}$ which is the $1 \mathrm{P}$ frequency ( 1 times the rotor frequency) for all three controllers. EOR and DAC show similar improvement over Baseline in the other parts of the spectrum. 


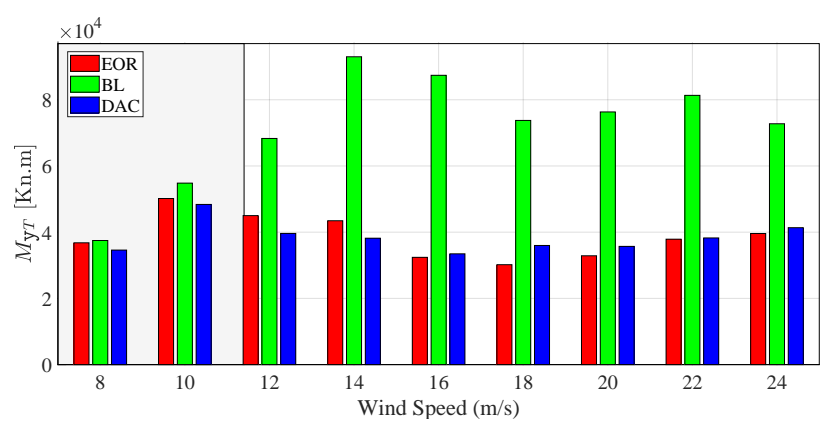

(a) Tower root fore-aft bending moment DEL

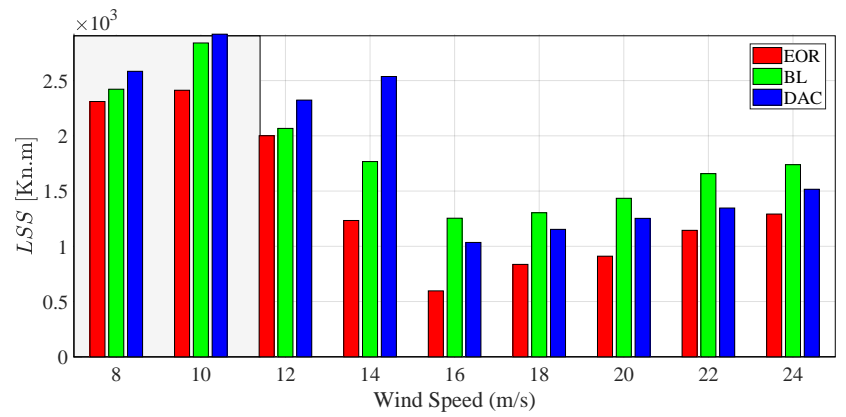

(c) Low speed shaft torsion DEL

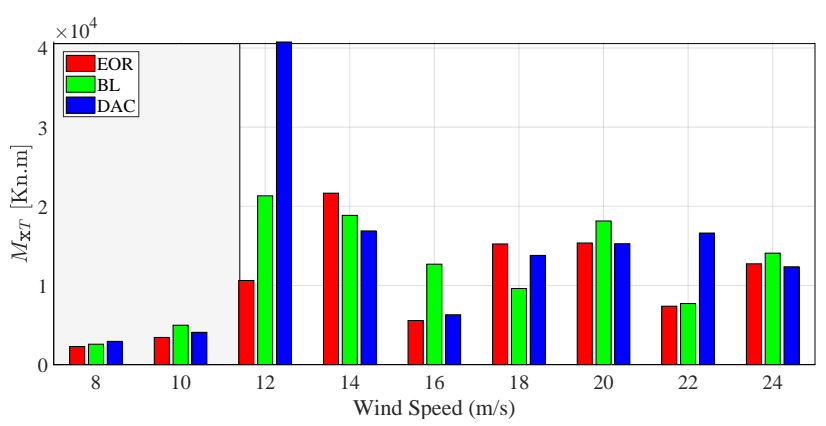

(b) Tower root side to side bending moment DEL

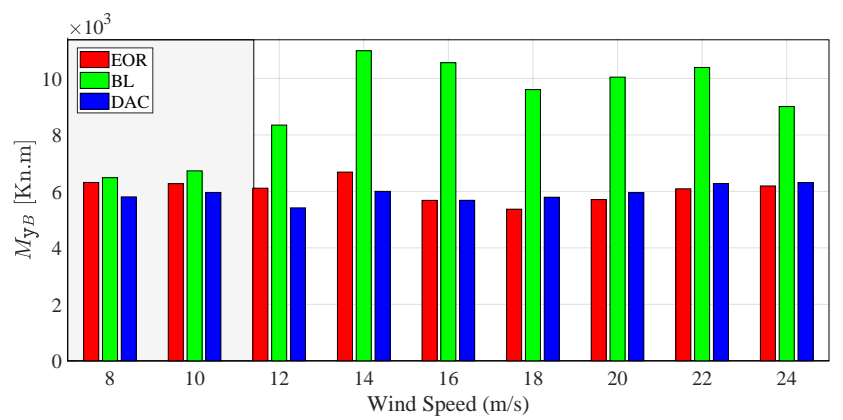

(d) Blade root flap-wise bending moment DEL

Figure 11: Damage equivalent loads for Class-A turbulent winds with mean wind speeds from $8 \mathrm{~m} / \mathrm{s}$ to $24 \mathrm{~m} / \mathrm{s}$.

Table 3: Weighted average of DEL and Power results for class A turbulent wind in both regions

\begin{tabular}{ccccccc}
\hline DELs \& STD & $M_{\mathrm{y} T}:[\mathrm{kNm}]$ & $M_{\mathrm{x} T}:[\mathrm{kNm}]$ & $M_{\mathrm{y} B}:[\mathrm{kNm}]$ & $M_{\mathrm{x} B}:[\mathrm{kNm}]$ & $L S S:[\mathrm{kNm}]$ & $P_{\text {mean }}:[\mathrm{MW}]$ \\
\hline \hline Baseline & $8.86 \mathrm{E}+04$ & $2.50 \mathrm{E}+04$ & $1.05 \mathrm{E}+04$ & $1.96+04$ & $2.59 \mathrm{E}+04$ & 3.75 \\
EOR & $5.20 \mathrm{E}+04$ & $1.98 \mathrm{E}+04$ & $6.68 \mathrm{E}+03$ & $1.95 \mathrm{E}+04$ & $2.28 \mathrm{E}+04$ & 3.74 \\
DAC & $5.72 \mathrm{E}+04$ & $3.66 \mathrm{E}+04$ & $6.07 \mathrm{E}+03$ & $1.95 \mathrm{E}+04$ & $2.72 \mathrm{E}+04$ & 3.74 \\
EOR $c f$. BL $\%$ & 41.3 & 20.8 & 33.5 & 0.5 & 13.1 & $\sim 0$ \\
DAC $c f$. BL $\%$ & 35.4 & -46.4 & 39.6 & 0.5 & -5.02 & $\sim 0$
\end{tabular}

\subsection{Power Generation Controller Performance Comparison}

To compare the power generation performance of the three controllers, we distinguish between Region 2 and Region 3 performance. Figures $14 \mathrm{a}$ and $14 \mathrm{~b}$ illustrate the standard deviations of rotor speed and generated power. Reducing these standard deviations implies less variation in these variables, indicating that the controller gives better performance in maintaining the rotor speeds and rated power at their rated values. These two graphs only contain Region 3 wind speeds, as these objectives only apply in Region 3 operation. Here EOR again outperforms both controllers, with the DAC controller giving significantly worse performance.

Table 4 summarizes the results of the controller performance for power generation. In Region 2, we have chosen a single wind speed of $9 \mathrm{~m} / \mathrm{s}$ to ensure the wind speed signal mostly remains above $7.85 \mathrm{~m} / \mathrm{s}$. For wind speeds below this level, the Baseline controller is not designed to track the optimal TSR, and this would invalidate the controller energy harvesting performance comparison. 


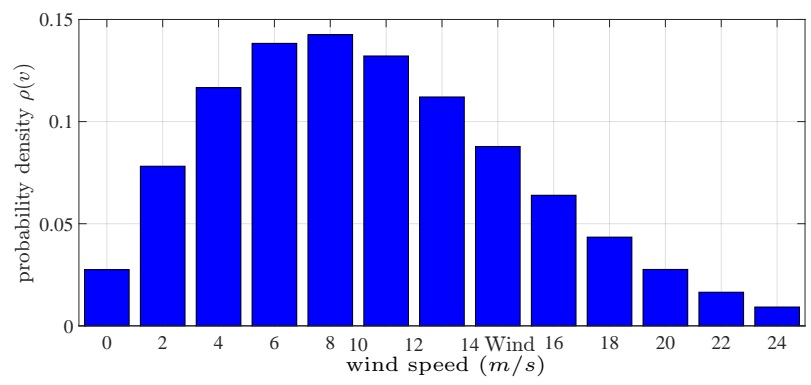

Figure 12: Weibull distribution of wind speed variation from measured data at a height of 102 $m$ in Bremerhaven [46].

Table 4: Power Generation Performance

\begin{tabular}{cccccc}
\hline & \multicolumn{2}{c}{ Region 2 $(9 \mathrm{~m} / \mathrm{sec})$} & \multicolumn{3}{c}{ Region 3 } \\
& $\operatorname{std}(\lambda)$ & $P:[\mathrm{MW}]$ & $\operatorname{std}\left(\Omega_{r}\right)$ & $\operatorname{std}(P)$ & $P:[\mathrm{MW}]$ \\
\hline \hline EOR & 0.534 & 2.15 & 0.034 & 153 & 4.71 \\
Baseline & 0.409 & 2.15 & 0.041 & 171 & 4.71 \\
DAC & 0.641 & 2.15 & 0.069 & 211 & 4.68 \\
EOR $c f$. BL $\%$ & -30.6 & $\sim 0$ & 17.9 & 10.5 & $\sim 0$ \\
DAC $c f$. BL $\%$ & -56.7 & $\sim 0$ & -67.1 & -23.4 & -0.6
\end{tabular}

The first column of the table shows that in Region 2, both EOR and DAC have considerably higher standard deviation in their TSR $\lambda$, indicating less rigid control of the rotor speed. While this might be expected to indicate a failure to achieve the optimal TSR for energy generation, column 2 of the table indicates negligible differences in the energy harvested by the three controllers. Thus the load reductions observed in Figures 11a to 11d have been achieved without sacrificing power generation.

The averaged results for the standard deviations $\operatorname{std}\left(\Omega_{r}\right)$ and $\operatorname{std}(P)$ are shown in columns 3 and 4 of Table 4 , with wind speeds weighted according to the Region 3 wind speeds in the Weibull distribution of Figure 12. The EOR controller achieved a substantial improvement in both rotor speed and power regulation by $17.9 \%$ and $10.5 \%$, relative to Baseline. Conversely, DAC suffered a performance degradation of $67.1 \%$ and $23.4 \%$, relative to Baseline. Column 5 of Table 4 show that EOR generated the same amount of power as Baseline, while DAC showed some very slight reduction in power generation.

Reducing rotor speed standard deviation in Region 3 reduces the likelihood that the rotor speed will violate the safe operational limits on $\Omega_{r}$. This reduces the chances of turbine failure and increases the turbine's operational availability.

\subsection{Pitch Actuation and Command Torque Rate}

The command torque rate (CTR) of the generator torque is given by

$$
C T R=\sqrt{\frac{1}{T} \int_{0}^{T}\left(\frac{d M_{g}(t)}{d t}\right)^{2} d t}
$$



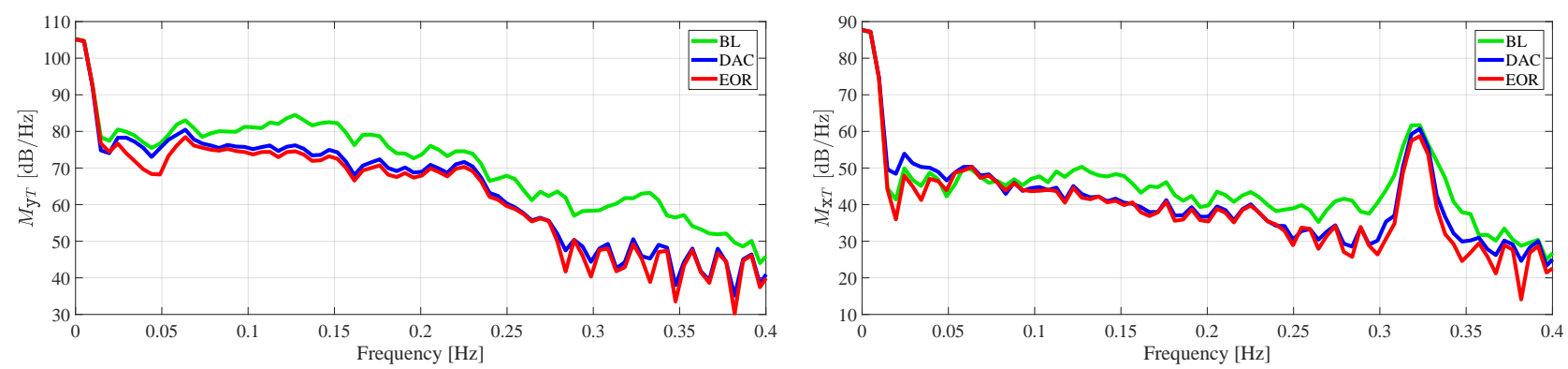

(a) PSD of Tower root fore-aft bending moment (b) PSD of Tower root side to side bending moment $M_{\mathrm{y} T}$ $M_{\mathrm{x} T}$
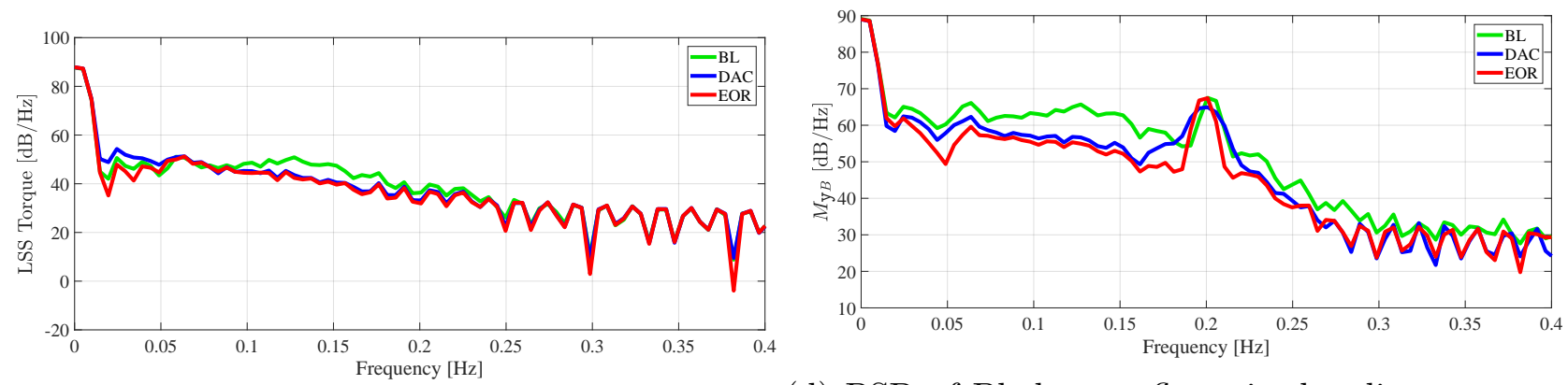

(c) PSD of Low speed shaft torsion $L S S$

(d) PSD of Blade root flap-wise bending moment $M_{\mathrm{y} B}$

Figure 13: Power Spectral Density Graphs of Loads at the wind speed $20 \mathrm{~m} / \mathrm{s}$.

The CTR measures changes to the generator torque set point during the 60 minute simulation period. From the system dynamic equations (6)-(7), we observe that changes in $M_{g}$ cause changes in the drive train torsion, and these variations are associated with fatigue on the drive shaft. Therefore, it is desirable for a controller to achieve its control objectives with reduced torque actuation. Figure 15 shows the CTR for Region 2 and 3 wind speeds.

The results show that EOR has the lowest CTR in all wind speeds except $12 \mathrm{~m} / \mathrm{s}$, indicating smoother torque control in both operating regions. DAC has lower CTR compared to Baseline except at wind speeds close to the transition region $(12$ to $14 \mathrm{~m} / \mathrm{s})$. The pitch travel $(\mathrm{PT})$ of the blade angle over the one-hour simulation period is defined to be

$$
P T=\int_{0}^{T}\left|\frac{d \theta}{d t}\right| d t
$$

Reducing pitch travel reduces wear and tear on the bearings of the pitch mechanism. Figure 16. shows the pitch travel for Region 3 wind speeds, where pitch actuation is active.

We observe that EOR and DAC have significantly lower pitch travel compared to Baseline. In higher than $16 \mathrm{~m} / \mathrm{s}$ wind speeds, EOR has slightly higher pitch travel than DAC. This may be related to the superior performance of EOR in controlling the rotor speed and output power at a more constant level, which requires greater pitch actuation.

\section{Conclusion and Future Work}

We have proposed a new strategy for wind turbine control using the classical EOR control methodology. Simulations were performed with the NREL FAST code for the NREL $5 \mathrm{MW}$ 


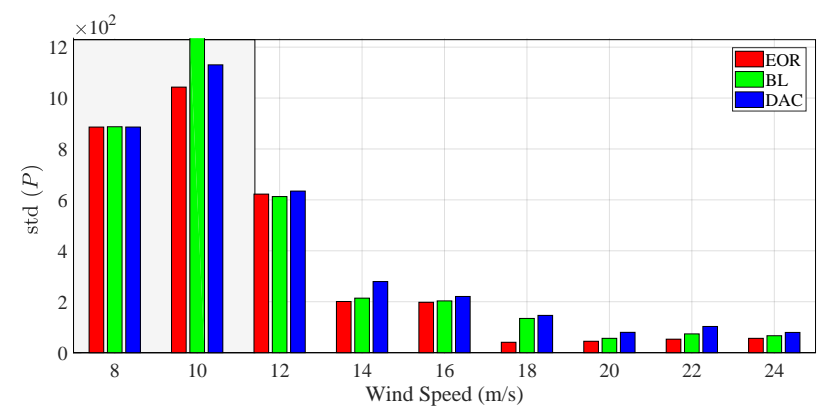

(a) Standard deviation of electric power generation

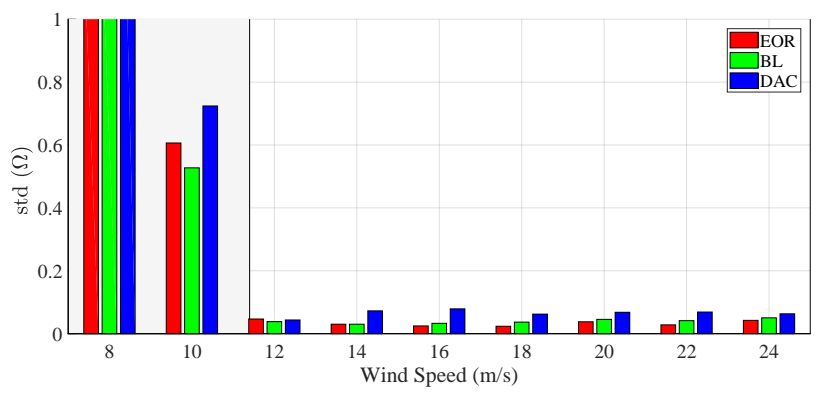

(b) Standard deviation of rotor speed

Figure 14: Standard deviation of power generation and rotor speed for Region 3 mean wind speeds

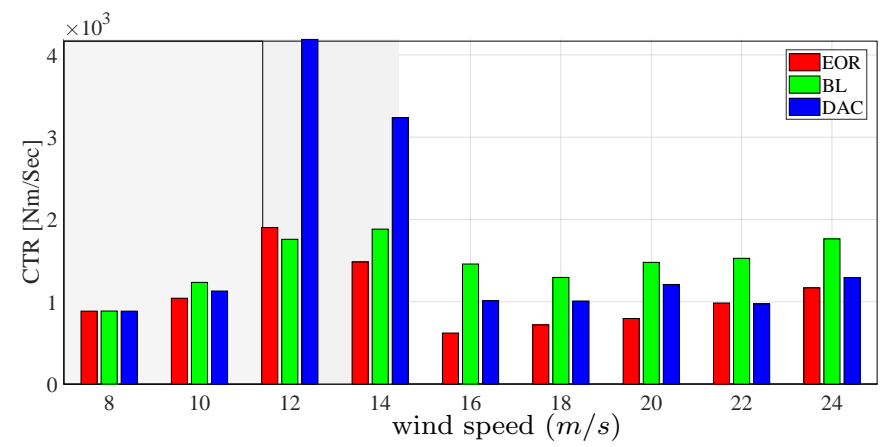

Figure 15: Command Torque Rate for Class-A turbulent winds with mean wind speeds from 8 to $24 \mathrm{~m} / \mathrm{s}$.

wind turbine model, using a broad range of realistic wind signals generated by TurbSim. Wind preview information is assumed to be available from LIDAR measurements. Our results showed the EOR controller was able to provide substantial and consistent fatigue load reductions compared to the Baseline controller and DAC, without loss of generated power, in both operating regions. Additionally, its modest computational cost means EOR can be expected to be suitable for real-time implementation.

In Region 2 maximizing the energy production requires (5) to hold at all times, and this requires the generator torque controller be designed to precisely track the optimal TSR, and all other states of the turbine to precisely track their equilibrium value for all instantaneous

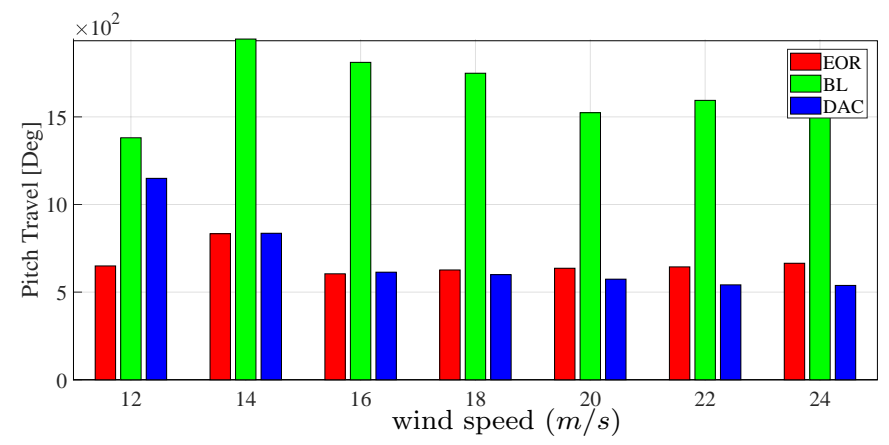

Figure 16: Pitch Travel in Region 3 for Class-A turbulent winds with mean wind speeds from $12 \mathrm{~m} / \mathrm{s}$ to $24 \mathrm{~m} / \mathrm{s}$. 
effective wind speeds. However, it was shown in [47] that the power coefficient $C_{p}$ is a relatively flat curve at its maximum and a controller designed to track the optimal TSR may contribute only very slight increases in power production, while adding a considerable amount of stress on the structure. Similar results were noted in [4], [48] and [47], where very small increases in power production came at the cost of substantial increases in DELs.

This situation suggests that proposed improvements in controller design methods should aim to reduce load fatigues, without compromising power harvesting efficiency. Our results in Region 2 showed that stress on the structure can be considerably reduced by EOR, without compromising energy capture. Similarly, our results in Region 3 showed that EOR can reduce variability in the rotor speed and power generation, again without compromising energy production.

Considering the relative performance of the EOR and DAC controllers, our performance comparisons showed an EOR controller was able to provide superior fatigue load reduction to a DAC controller. The feedforward gain used in DAC does not take the wind dynamics into account, and this a restricting assumption when dealing with rapidly varying disturbances. Conversely, EOR accommodates higher-order dynamics when modelling wind disturbances, by constructing an exosystem whose states include derivatives of the disturbance. Therefore, EOR is better able to utilise the wind information obtained from LIDAR to develop wind prediction capability, by utilising time derivatives of the disturbance signal. However, our study does not allow for wind evolution between the LIDAR focal point and the blades, nor does it consider LIDAR measurement noise. The presence of either of these factors can be expected to reduce the observed performance improvements of both EOR and DAC over Baseline.

The order of the exosystem employed within the EOR methodology can be freely chosen depending on the reliability of the LIDAR measurements, computational power and the parameter estimation limitations. Therefore, it is possible to design the EOR controller with regard to different control performance objectives. For example, a higher-order exosystem will yield a more precise description of the wind and may improve disturbance rejection. However, it may also increase the structural loads and actuation efforts. Hence, selecting the appropriate order for the exosystem involves a trade-off between increasing the power generation performance and reducing the loads.

The classical EOR controller design method described in section 3.2 has been extended to accommodate other control problem frameworks, such as the robust output regulation problem, in which the objective is to achieve output regulation in the presence of plant uncertainty, and the nonlinear output regulation problem which considers the problem of regulating the output of a nonlinear plant [49]. Both of these variations on EOR have the potential to further improve turbine performance, by accommodating the plant uncertainty introduced by use of the linearised model (12), or else through the direct use of the nonlinear model in (7)-(9) for the controller design.

Future developments will also consider the performance of the EOR methodology using individual pitch control. It is anticipated this yield further improvements in rotor speed control with reduced fatigue loads, in comparison with Baseline and DAC.

Another area for future work is to develop a transition strategy between Regions 2 and 3. For mean wind speeds near the transition wind speed of $11.4 \mathrm{~m} / \mathrm{s}$, our analysis showed EOR gave reduced improvements than when the mean wind speeds were not close to transition. For instance, standard deviation of rotor speed $\operatorname{std}\left(\Omega_{r}\right)$ at the reference wind speed of $12 \mathrm{~m} / \mathrm{s}$ is higher under EOR than both Baseline and DAC, in contrast with the superior performance of EOR at all other Region 3 wind speeds. Similarly, the command torque ratio at $12 \mathrm{~m} / \mathrm{s}$ for 
EOR is higher than Baseline which can clearly be associated with transition region issues. The EOR performance is clearly better than Baseline at all other mean wind speeds. Therefore, an EOR transition strategy is needed to enable more effective switching from generator torque control to blade pitch control as wind speeds vary between Region 2 and 3.

\section{Acknowledgment}

The authors would like to thank D. Schlipf and M. Mirzaei for numerous helpful suggestions, and the anonymous reviewers for their detailed and insightful comments.

\section{References}

[1] Z. Chen, K. Stol, and B. Mace, "Wind turbine blade optimisation with individual pitch and trailing edge flap control," Renewable energy, vol. 103, pp. 750-765, 2017.

[2] E. Muljadi and C. P. Butterfield, "Pitch-controlled variable-speed wind turbine generation," IEEE Transactions on Industry Applications, vol. 37, no. 1, pp. 240-246, 2001.

[3] M. Harris, M. Hand, and A. Wright, "Lidar for turbine control," National Renewable Energy Laboratory, Golden, CO, Report No. NREL/TP-500-39154, 2006.

[4] N. Wang, K. E. Johnson, and A. D. Wright, "Comparison of strategies for enhancing energy capture and reducing loads using lidar and feedforward control," IEEE Transactions on Control Systems Technology, vol. 21, no. 4, pp. 1129-1142, 2013.

[5] A. Scholbrock, P. Fleming, D. Schlipf, A. Wright, K. Johnson, and N. Wang, "Lidarenhanced wind turbine control: Past, present, and future," in American Control Conference $(A C C)$, 2016. IEEE, 2016, pp. 1399-1406.

[6] J.P. Goit, S. Shimada, and T. Kogaki, "Can LIDARs Replace Meteorological Masts in Wind Energy?" Energies, 2019, 12, 3680.

[7] D. Schlipf and M. Kühn, "Prospects of a collective pitch control by means of predictive disturbance compensation assisted by wind speed measurements," in Proceedings of the 9th German Wind Energy Conference DEWEK, 2008.

[8] F. Dunne, L. Y. Pao, A. D. Wright, B. Jonkman, and N. Kelley, "Adding feedforward blade pitch control to standard feedback controllers for load mitigation in wind turbines," Mechatronics, vol. 21, no. 4, pp. 682-690, 2011.

[9] J. Laks, L. Y. Pao, A. Wright, N. Kelley, and B. Jonkman, "Blade pitch control with preview wind measurements," in Proceedings of the 48th AIAA Aerospace Sciences Meeting Including the New Horizons Forum and Aerospace Exposition, 2010.

[10] F. Dunne, L. Y. Pao, A. D. Wright, B. Jonkman, and N. Kelley, "Combining standard feedback controllers with feedforward blade pitch control for load mitigation in wind turbines," in Proc. 48th AIAA Aerospace Sciences Meeting, Orlando, FL, AIAA-2010-250, 2010 . 
[11] A. Scholbrock, P. Fleming, L. Fingersh, A. Wright, D. Schlipf, F. Haizmann, F. Belen et al., "Field testing lidar based feed-forward controls on the nrel controls advanced research turbine," in 51th AIAA Aerospace Sciences Meeting Including the New Horizons Forum and Aerospace Exposition, 2013.

[12] D. Schlipf, P. Fleming, F. Haizmann, A. Scholbrock, M. Hofsäß, A. Wright, and P. W. Cheng, "Field testing of feedforward collective pitch control on the cart2 using a nacellebased lidar scanner," in Journal of Physics: Conference Series, vol. 555, no. 1. IOP Publishing, 2014, p. 012090.

[13] F. Haizmann, D. Schlipf, S. Raach, A. Scholbrock, A. Wright, C. Slinger, J. Medley, M. Harris, E. Bossanyi, and P. W. Cheng, "Optimization of a feed-forward controller using a CW-lidar system on the CART3," in American Control Conference (ACC), 2015. IEEE, 2015, pp. 3715-3720.

[14] D. Schlipf, S. Kapp, J. Anger, O. Bischoff, M. Hofsäß, A. Rettenmeier, and M. Kühn, "Prospects of optimization of energy production by lidar assisted control of wind turbines," in EWEA proceedings, 2011.

[15] D. Schlipf, P. Fleming, S. Kapp, A. Scholbrock, F. Haizmann, F. Belen, A. Wright, and P. W. Cheng, "Direct speed control using lidar and turbine data," in American Control Conference (ACC), 2013. IEEE, 2013, pp. 2208-2213.

[16] K. E. Johnson, L. Y. Pao, M. J. Balas, and L. J. Fingersh, "Control of variable-speed wind turbines: standard and adaptive techniques for maximizing energy capture," IEEE control systems, vol. 26, no. 3, pp. 70-81, 2006.

[17] K. Takaba, "A tutorial on preview control systems," in SICE 2003 Annual Conference, vol. 2. IEEE, 2003, pp. 1388-1393.

[18] K. A. Stol, "Disturbance tracking and blade load control of wind turbines in variable-speed operation," in Proceedings of the 22nd AIAA/ASME Wind Energy Symposium, 2003, pp. $317-323$.

[19] M. Balas, Y. Lee, and L. Kendall, "Disturbance tracking control theory with application to horizontal axis wind turbines," in 1998 ASME Wind Energy Symposium, 1998, p. 32.

[20] A. D. Wright, "Modern control design for flexible wind turbines," National Renewable Energy Laboratory, Golden, CO (US), Tech. Rep., 2004.

[21] M. M. Hand, K. E. Johnson, L. Fingersh, and A. D. Wright, "Advanced control design and field testing for wind turbines at the national renewable energy laboratory," National Renewable Energy Laboratory, Golden, CO, NREL Technical Report No. CP-500-36118, 2004.

[22] K. A. Stol and M. J. Balas, "Periodic disturbance accommodating control for blade load mitigation in wind turbines," Journal of Solar Energy Engineering, vol. 125, no. 4, pp. 379-385, 2003.

[23] A. D. Wright, L. Fingersh, and M. Balas, "Testing state-space controls for the controls advanced research turbine: Preprint," National Renewable Energy Lab.(NREL), Golden, CO (United States), Tech. Rep., 2006. 
[24] D. Schlipf, D. J. Schlipf, and M. Kühn, "Nonlinear model predictive control of wind turbines using lidar," Wind Energy, vol. 16, no. 7, pp. 1107-1129, 2013.

[25] E. Tofighi, D. Schlipf, and C. M. Kellett, "Nonlinear model predictive controller design for extreme load mitigation in transition operation region in wind turbines," in Control Applications (CCA), 2015 IEEE Conference on. IEEE, 2015, pp. 1167-1172.

[26] A. Mahdizadeh, E. Tofighi, and R. Schmid, "Comparison between the nmpc and eor control of wind turbines using LIDAR wind measurements," in Journal of Physics: Conference Series, vol. 1037, no. 3. IOP Publishing, 2018, p. 032046.

[27] H. L. Trentelman, A. A. Stoorvogel, and M. Hautus, "Control theory for linear systems," Communications and Control Engineering Series, Springer, 2002.

[28] A. Saberi, A. A. Stoorvogel, and P. Sannuti, Control of linear systems with regulation and input constraints. Springer Science \& Business Media, 2000.

[29] A. Mahdizadeh, R. Schmid, and D. Oetomo, "Enhanced Energy Capture of Wind Turbines by Exact Output Regulation," in Decision and Control (CDC), 2017 IEEE 56th Annual Conference on. IEEE, 2017, pp. 1446-1451.

[30] A. Mahdizadeh, R. Schmid, and D. Oetomo, "Fatigue Load Mitigation in Multi-megawatt Wind Turbines Using Output Regulation Control," in 2017 21st International Conference on System Theory, Control and Computing (ICSTCC). IEEE, 2017, pp. 163-168.

[31] J. Jonkman, S. Butterfield, W. Musial, and G. Scott, "Definition of a 5-MW reference wind turbine for offshore system development," National Renewable Energy Laboratory (NREL), Golden, CO., Tech. Rep., 2009.

[32] J. M. Jonkman, M. L. Buhl Jr et al., "FAST users guide," National Renewable Energy Laboratory, Golden, CO, Technical Report No. NREL/EL-500-38230, 2005.

[33] B. J. Jonkman, "TurbSim user's guide: Version 1.50," National Renewable Energy Laboratory, Colorado, Tech. Rep., 2009.

[34] A. Nieslony, "Rainflow counting algorithm, version 1.2," http://au.mathworks.com/matlabcentral/fileexchange/3026-rainflow-counting-algorithm, Apr. 2010.

[35] G. A. Van Kuik, "The Lanchester-Betz-Joukowsky limit," Wind Energy, vol. 10, no. 3, pp. 289-291, 2007.

[36] M. Mirzaei, "Wind turbine control: Robust model based approach," Ph.D. dissertation, Technical University of Denmark, 2012.

[37] T. Ono and J. Arai, "Frequency control with dead band characteristic of battery energy storage system for power system including large amount of wind power generation," IEEJ Transactions on Power and Energy, vol. 132, pp. 709-717, 2012.

[38] K. Yamashita, O. Sakamoto, Y. Kitauchi, T. Nanahara, T. Inoue, T. Shiohama, and H. Fukuda, "Development of frequency stabilizing scheme for integrating wind power generation into an isolated grid," IEEJ Transactions on Power and Energy, vol. 131, pp. 437-446, 2011. 
[39] R. Schmid and L. Ntogramatzidis, "Nonovershooting and nonundershooting exact output regulation," Systems \& Control Letters, vol. 70, pp. 30-37, 2014.

[40] A. D. Wright and L. Fingersh, "Advanced control design for wind turbines; part i: control design, implementation, and initial tests," National Renewable Energy Laboratory (NREL), Golden, CO., Tech. Rep., 2008.

[41] M. Balas, K. Thapa Magar, and S. Frost, "Adaptive tracking control of partially known signals with application to large wind turbines in region 2 operation," in Infotech@ Aerospace 2011, 2011, p. 1429.

[42] F. Dunne, E. Simley, and L. Pao, "Lidar wind speed measurement analysis and feedforward blade pitch control for load mitigation in wind turbines," National Renewable Energy Laboratory, Golden, CO, 2011.

[43] N. D. Kelley, "A case for including atmospheric thermodynamic variables in wind turbine fatigue loading parameter identification," National Renewable Energy Laboratory, Golden, CO (US), Tech. Rep., 1999.

[44] IEC Std., Rev., IEC 61400-1 third edition 2005-08 Wind turbines - Part 1: Design requirements. IEC Std., Rev., 2005.

[45] S. D. Downing and D. Socie, "Simple rainflow counting algorithms," International Journal of Fatigue, vol. 4, no. 1, pp. 31-40, 1982.

[46] D. Schlipf, "Lidar-assisted control concepts for wind turbines," Ph.D. dissertation, University of Stuttgart, 2016.

[47] E. Bossanyi, A. Kumar, and O. Hugues-Salas, "Wind turbine control applications of turbine-mounted LIDAR," in Journal of Physics: Conference Series, vol. 555, no. 1. IOP Publishing, 2014, p. 012011.

[48] N. Wang, K. E. Johnson, A. D. Wright, and C. E. Carcangiu, "Lidar-assisted wind turbine feedforward torque controller design below rated," in American Control Conference (ACC), 2014. IEEE, 2014, pp. 3728-3733.

[49] J. Huang, Nonlinear Output Regulation: Theory and Applications. SIAM, 2004, vol. 8. 